\title{
Optimal Smoothing for a Computationally and Statistically Efficient Single Index Estimator
}

\author{
Yingcun Xia \\ (National University of Singapore) \\ Wolfgang Härdle \\ (Humboldt-Universität zu Berlin)
}

Oliver Linton

(London School of Economics)

DP No: EM 2009537

The Suntory Centre

Suntory and Toyota International Centres for

Economics and Related Disciplines

London School of Economics and Political Science

Houghton Street

London WC2A 2AE

2009

Tel: 02079556674 


\begin{abstract}
In semiparametric models it is a common approach to under-smooth the nonparametric functions in order that estimators of the finite dimensional parameters can achieve root- $n$ consistency. The requirement of under-smoothing may result as we show from inefficient estimation methods or technical difficulties. Based on local linear kernel smoother, we propose an estimation method to estimate the single-index model without under-smoothing. Under some conditions, our estimator of the single-index is asymptotically normal and most efficient in the semi-parametric sense. Moreover, we derive higher expansions for our estimator and use them to define an optimal bandwidth for the purposes of index estimation. As a result we obtain a practically more relevant method and we show its superior performance in a variety of applications.
\end{abstract}

Key words and phrases: ADE; Asymptotics; Bandwidth; MAVE method; Semiparametric efficiency.

The authors. All rights reserved. Short sections of text, not to exceed two paragraphs, may be quoted without explicit permission provided that full credit, including $\odot$ notice, is given to the source. 


\section{Introduction}

Single index models (SIMs) are widely used in the applied quantitative sciences. Although the context of applications for SIMs almost never prescribes the functional or distributional form of the involved statistical error, the SIM is commonly fitted with (low dimensional) likelihood principles. Both from a theoretical and practical point of view such fitting approach has been criticized and has led to semiparametric modelling. This approach involves high dimensional parameters (nonparametric functions) and a finite dimensional index parameter. Consider the following single-index model,

$$
Y=g\left(\theta_{0}^{\top} X\right)+\varepsilon
$$

where $E(\varepsilon \mid X)=0$ almost surely, $g$ is an unknown link function, and $\theta_{0}$ is a single-index parameter with length one and first element positive for identification. In this model there is a single linear combination of covariates $X$ that can capture most information about the relation between response variable $Y$ and covariates $X$, thereby avoiding the "curse of dimensionality". Estimation of the single-index model is very attractive both in theory and in practice. In the last decade a series of papers has considered estimation of the parametric index and the nonparametric part with focus on root-n estimability and efficiency issues, see Carroll, Fan, Gijbels and Wand (1997) for an overview. There are numerous methods proposed or can be used for the estimation of the model. Amongst them, the most popular ones are the average derivative estimation (ADE) method investigated by Härdle and Stoker (1989), the sliced inverse regression (SIR) method proposed by Li (1989); the semiparametric least squares (SLS) method of Ichimura (1993) and the simultaneous minimization method of Härdle, Hall and Ichimura (1993).

The existing estimation methods are all subject to some or other of the following four critiques: (1) Heavy computational burden: see, for example, Härdle, Hall and Ichimura (1993), Delecroix, Härdle and Hristache (2003), Xia and Li (1999) and Xia et al. (1999). These methods include complicated optimization techniques (iteration between bandwidth choice and parameter estimation) for which no simple and effective algorithm is available up to now. (2) Strong restrictions on link functions or design of covariates $X$ : $\mathrm{Li}$ (1991) required the covariate to have a symmetric distribution; Härdle and Stoker (1989) and Hristache et al. (2001) needed a non-symmetric structure for the link function, i.e., $\left|E g^{\prime}\left(\theta_{0}^{\top} X\right)\right|$ is bounded away from 0. If these conditions are violated, the corresponding methods are inconsistent. (3) Inefficiency: The ADE method of Härdle and Stoker (1989) or the improved ADE method of Hristache et al. (2001) is not asymptotically efficient in the semi-parametric sense, Bickel et al. (1993). Nishiyama and Robinson (2000, 
2005) considered the Edgeworth correction to the ADE methods. Härdle and Tsybakov (1993) discussed the sensitivity of the ADE. Since this method involves high dimensional smoothing and derivative estimation, its higher order properties are poor. (4) Under-smoothing: Let $h_{g}^{o p t}$ be the optimal bandwidth in the sense of MISE for the estimation of link function $g$ and let $h_{\theta}$ be the bandwidth used for the estimation of $\theta_{0}$. Most of the methods mentioned above require the bandwidth $h_{\theta}$ to be much smaller than the bandwidth $h_{g}^{\text {opt }}$, i.e. $h_{\theta} / h_{g}^{o p t} \rightarrow 0$ as $n \rightarrow \infty$, in order that estimators of $\theta_{0}$ can achieve root- $n$ consistency, see, Härdle, and Stoker (1989) and Hristache et al. (2002), Robinson (1988), Hall (1989) and Carroll et al. (1997) among others. Due to technical complexities, there are few investigations about how to select the bandwidth $h_{\theta}$ for the estimation of the single-index. Thus it could be the case that even if $h_{\theta}=h_{g}^{\text {opt }}$ allows for root-n consistent estimation of $\theta$, that $h_{\theta}^{o p t} / h_{g}^{o p t} \rightarrow 0$ or $h_{g}^{o p t} / h_{\theta}^{o p t} \rightarrow 0$, where $h_{\theta}^{o p t}$ is the optimal bandwidth for estimation of $\theta$. This would mean that using a single bandwidth $h_{g}^{\text {opt }}$ would result in suboptimal performance for the estimator of $\theta$. Higher order properties of other semiparametric procedures have been studied in Linton (1995) inter alia.

Because the estimation of $\theta_{0}$ is based on the estimation of the link function $g$, we might expect that a good bandwidth for the link function should be a good bandwidth for the single-index, i.e., under-smoothing should be unnecessary. Unfortunately, most of the existing estimation methods involve for technical reason "under-smoothing" the link function in order to obtain a root- $n$ consistent estimator of $\theta_{0}$. See, for example, Härdle and Stoker (1989), Hristache et al. (2001, 2002), Carroll et al. (1997) and Xia and Li (1999). Härdle, Hall and Ichimura (1993) investigated this problem for the first time and proved that the optimal bandwidth for the estimation of the link function in the sense of MISE can be used for the estimation of the single-index to achieve root- $n$ consistency. As mentioned above, for its computational complexity the method of Härdle, Hall and Ichimura (1993) is hard to implement in practice.

This paper presents a method of joint estimation of the parametric and nonparametric parts. It avoids undersmoothing and the computational complexity of former procedures and achieves the semiparametric efficiency bound. It is based on the MAVE method of Xia et al (2002), which we outline in the next section. Using local linear approximation and global minimization, we give a very simple iterative algorithm. The proposed method has the following advantages: (i) the algorithm only involves one-dimensional smoothing and is proved to converge at a geometric rate; (ii) with normal errors in the model, the estimator of $\theta_{0}$ is asymptotically normal and efficient in the semiparametric sense; (iii) the optimal bandwidth for the estimation of the link function in the sense of MISE can be used to estimate $\theta_{0}$ with root- $n$ consistency; 
(iv) by a second order expansion, we further show that the optimal bandwidth for the estimation of the single-index $\theta_{0}, h_{\theta}^{o p t}$, is of the same magnitude as $h_{g}^{o p t}$.

Therefore, the commonly used "under-smoothing" approach is inefficient in the sense of second order approximation. Powell and Stoker (1996) investigated bandwidth selection for the ADE methods. We also propose an automatic bandwidth selection method for our estimator of $\theta$. Xia (2006) has recently shown the first order asymptotic properties of this method. Our theoretical results are proven under weak moment conditions.

In section 3 we present our main results. We show the speed of convergence, give the asymptotic estimation and derive a smoothing parameter selection procedure. In the following section we investigate the proposed estimator in simulation and application. Technical details are deferred to the appendix.

\section{The MAVE method}

Suppose that $\left\{X_{i}, Y_{i}: i=1,2, \ldots, n\right\}$ is a random sample from model (1). The basic idea of our estimation method is to linearly approximate the smooth link function $g$ and to estimate $\theta_{0}$ by minimizing the overall approximation errors. Xia et al (2002) proposed a procedure via the so called minimum average conditional variance estimation (MAVE). The single index model (11) is a special case of what they considered, and we can estimate it as follows. Assuming function $g$ and parameter $\theta_{0}$ are known, then the Taylor expansion of $g\left(\theta_{0}^{\top} X_{i}\right)$ at $g\left(\theta_{0}^{\top} x\right)$ is

$$
g\left(\theta_{0}^{\top} X_{i}\right) \approx a+d \theta_{0}^{\top}\left(X_{i}-x\right)
$$

where $a=g\left(\theta_{0}^{\top} x\right)$ and $d=g^{\prime}\left(\theta_{0}^{\top} x\right)$. With fixed $\theta$, the local estimator of the conditional variance is then

$$
\sigma_{n}^{2}(x \mid \theta)=\min _{a, d}\left\{n \hat{f}_{\theta}(x)\right\}^{-1} \sum_{i=1}^{n}\left[Y_{i}-\left\{a+d \theta^{\top}\left(X_{i}-x\right)\right\}\right]^{2} K_{h}\left\{\theta^{\top}\left(X_{i}-x\right)\right\},
$$

where $\hat{f}_{\theta}(x)=n^{-1} \sum_{i=1}^{n} K_{h}\left\{\theta^{\top}\left(X_{i}-x\right)\right\}$, where $K$ is a univariate density function, $h$ is the bandwidth and $K_{h}(u)=K(u / h) / h$; see Fan et al (1996). The value $\sigma_{n}^{2}(x \mid \theta)$ can also be understood as the local departure of $Y_{i}$ with $X_{i}$ close to $x$ from a local linear model with given $\theta$. Obviously, the best approximation of $\theta$ should minimize the overall departure at all $x=X_{j}, j=1, \cdots, n$. Thus, our estimator of $\theta_{0}$ is to minimize

$$
Q_{n}(\theta)=\sum_{j=1}^{n} \sigma_{n}^{2}\left(X_{j} \mid \theta\right)
$$


with respect to $\theta:|\theta|=1$. This is the so-called minimum average conditional variance estimation (MAVE) in Xia et al (2002). In practice it is necessary to include some trimming in covariate regions where density is low, so we weight $\sigma_{n}^{2}\left(X_{j} \mid \theta\right)$ by a sequence $\hat{\rho}_{j}^{\theta}$, where $\hat{\rho}_{j}^{\theta}=\rho_{n}\left\{\hat{f}_{\theta}\left(X_{j}\right)\right\}$, that is discussed further below.

The corresponding algorithm can be stated as follows. Suppose $\theta_{1}$ is an initial estimate of $\theta_{0}$. Set the number iteration $\tau=1$ and bandwidth $h_{1}$. We also set a final bandwidth $h$. Let $X_{i j}=X_{i}-X_{j}$.

Step 1: With bandwidth $h_{\tau}$, calculate $\hat{f}_{\theta}\left(X_{j}\right)=n^{-1} \sum_{i=1}^{n} K_{h_{\tau}}\left(\theta^{\top} X_{i j}\right)$ and the solutions of $a_{j}$ and $d_{j}$ to the inner problem in (2)

$$
\left(\begin{array}{c}
a_{j}^{\theta} \\
d_{j}^{\theta} h_{\tau}
\end{array}\right)=\left\{\sum_{i=1}^{n} K_{h_{\tau}}\left(\theta^{\top} X_{i j}\right)\left(\begin{array}{c}
1 \\
\theta^{\top} X_{i j} / h_{\tau}
\end{array}\right)\left(\begin{array}{c}
1 \\
\theta^{\top} X_{i j} / h_{\tau}
\end{array}\right)^{\top}\right\}^{-1} \sum_{i=1}^{n} K_{h_{\tau}}\left(\theta^{\top} X_{i j}\right)\left(\begin{array}{c}
1 \\
\theta^{\top} X_{i j} / h_{\tau}
\end{array}\right) Y_{i} .
$$

Step 2: Fix the weight $K_{h_{\tau}}\left(\theta^{\top} X_{i j}\right), f_{\theta}\left(X_{j}\right), a_{j}^{\theta}$ and $d_{j}^{\theta}$. Calculate the solution of $\theta$ to (2)

$$
\theta=\left\{\sum_{i, j=1}^{n} K_{h_{\tau}}\left(\theta^{\top} X_{i j}\right) \hat{\rho}_{j}^{\theta}\left\{d_{\theta}\left(X_{j}\right)\right\}^{2} X_{i j} X_{i j}^{\top} \hat{f}_{\theta}\left(\theta^{\top} X_{j}\right)\right\}^{-1} \sum_{i, j=1}^{n} K_{h_{\tau}}\left(\theta^{\top} X_{i j}\right) \hat{\rho}_{j}^{\theta} d_{\theta}\left(X_{j}\right) X_{i j}\left(y_{i}-a_{j}^{\theta}\right) / \hat{f}_{\theta}\left(\theta^{\top} X_{j}\right),
$$

where $\hat{\rho}_{j}^{\theta}=\rho_{n}\left\{\hat{f}_{\theta}\left(X_{j}\right)\right\}$.

Step 3: Set $\tau=\tau+1, \theta:=\theta /|\theta|$ and $h_{\tau}:=\max \left\{h, h_{\tau} / \sqrt{2}\right\}$, go to Step 1 .

Repeat steps 1 and 2 until convergence.

The iteration can be stopped by the common rule. For example, if the calculated $\theta$ 's are stable at a certain direction, we can stop the iteration. The final vector $\theta:=\theta /|\theta|$ is the MAVE estimator of $\theta_{0}$, denoted by $\hat{\theta}$. Note that these steps are an explicit algorithm of the Xia et al (2002) method for the single-index model with some version of what the called 'refined kernel weighting' and boundary trimming. Similar to the other direct estimation methods, the calculation above is easy to implement. See Horowitz and Härdle (1996) for more discussions. After $\theta$ is estimated, the link function can be then estimated by the local linear smoother as $g^{\hat{\theta}}(v)$, where

$$
\hat{g}^{\theta}(v)=\left[n\left\{s_{2}^{\theta}(v) s_{0}^{\theta}(v)-\left(s_{1}^{\theta}(v)\right)^{2}\right\}\right]^{-1} \sum_{i=1}^{n}\left\{s_{2}^{\theta}(v)-s_{1}^{\theta}(v)\left(\theta^{\top} X_{i}-v\right) / h_{\tau}\right\} K_{h_{\tau}}\left(\theta^{\top} X_{i}-v\right) Y_{i},
$$

and $s_{k}^{\theta}(v)=n^{-1} \sum_{i=1}^{n} K_{h_{\tau}}\left(\theta^{\top} X_{i}-v\right)\left\{\left(\theta^{\top} X_{i}-v\right) / h_{\tau}\right\}^{k}$ for $k=0,1,2$. Actually, $\hat{g}^{\hat{\theta}}(v)$ is the final value of $a_{j}^{\theta}$ in Step 1 with $\theta^{\top} X_{j}$ replaced by $v$.

In the algorithm, $\rho_{n}($.$) is a trimming function employed to handle the boundary points. There are many$ choices for the estimator to achieve the root- $n$ consistency; see e.g. Härdle and Stocker (1989) and HHI 
(1993). However, to achieve the efficiency bound, $\rho_{n}(v)$ must tend to 1 for all $v$. In this paper, we take $\rho_{n}(v)$ as a bounded function with third order derivatives on $\mathbb{R}$ such that $\rho_{n}(v)=1$ if $v>2 c_{0} n^{-\varsigma} ; \rho_{n}(v)=0$ if $v \leq c_{0} n^{-\varsigma}$ for some constants $\varsigma>0$ and $c_{0}>0$. As an example, we can take

$$
\rho_{n}(v)= \begin{cases}1, & \text { if } v \geq 2 c_{0} n^{-\varsigma} \\ \frac{\exp \left\{\left(2 c_{0} n^{-\varsigma}-v\right)^{-1}\right\}}{\exp \left\{\left(2 c_{0} n^{-\varsigma}-v\right)^{-1}\right\}+\exp \left\{\left(v-c_{0} n^{-\varsigma}\right)^{-1}\right\}}, & \text { if } 2 c_{0} n^{-\varsigma}>v>c_{0} n^{-\varsigma} \\ 0, & \text { if } v \leq c_{0} n^{-\varsigma}\end{cases}
$$

The choice of $\varsigma$ will be given below.

\section{Main Results}

We impose the following conditions to obtain the asymptotics of the estimators.

[(C1)] [Initial estimator] The initial estimator is in $\Theta_{n}=\left\{\theta:\left|\theta-\theta_{0}\right| \leq n^{-\alpha}\right\}$ for some $0<\alpha \leq 1 / 2$.

[(C2)] [Design] The density function $f_{\theta}(v)$ of $\theta^{\top} X$ and its derivatives up to 6th order are bounded on $\mathbb{R}$ for all $\theta \in \Theta_{n}, E|X|^{6}<\infty$ and $E|Y|^{3}<\infty$. Furthermore, $\sup _{v \in R, \theta \in \Theta_{n}}\left|f_{\theta}(v)-f_{\theta_{0}}(v)\right| \leq c\left|\theta-\theta_{0}\right|$ for some constant $c>0$.

[(C3)] [Link function] The conditional mean $g_{\theta}(v)=E\left(Y \mid \theta^{\top} X=v\right), E\left(X \mid \theta^{\top} X=v\right), E\left(X X^{\top} \mid \theta^{\top} X=v\right)$ and their derivatives up to 6th order are bounded for all $\theta:\left|\theta-\theta_{0}\right|<\delta$ where $\delta>0$.

[(C4)] [Kernel function] $K(v)$ is a symmetric density function with finite moments of all orders.

[(C5)] [Bandwidth and trimming parameter] Trimming parameter $\varsigma \leq 1 / 20$ and bandwidth $h \propto n^{-\rho}$ for some $\rho$ with $1 / 5-\epsilon \leq \rho \leq 1 / 5+\epsilon$ for some $\epsilon>0$.

Assumption (C1) is feasible because such an initial estimate is obtainable using existing methods, such as Härdle and Stoker (1989), Powell et al. (1989) and Horowitz and Härdle (1996). Actually, Härdle, Hall and Ichimura (1993) even assumed that the initial value is in a root- $n$ neighborhood of $\theta_{0},\left\{\theta:\left|\theta-\theta_{0}\right| \leq C_{0} n^{-1 / 2}\right\}$. Assumption (C2) means that $X$ may have discrete components providing that $\theta^{\top} X$ is continuous for $\theta$ in a small neighborhood of $\theta_{0}$; see also Ichimura (1993). The moment requirement on $X$ is not strong. Härdle, Hall and Ichimura (1993) obtained their estimator in a bounded area of $\mathbb{R}^{p}$, which is equivalent to assume that $X$ is bounded; see also Härdle and Stoker (1989). We impose slightly higher order moment requirement than second moment for $Y$ to ensure the optimal bandwidth in (C5) can be used in applying Lemma 6.1 in 
section 6. The smoothness requirements on the link function in (C3) can be relaxed to the existence of a bounded second order derivative at the cost of more complicated proofs and smaller bandwidth. Assumption (C4) includes the Gaussian kernel and the quadratic kernel. Assumption (C5) includes the commonly used optimal bandwidth in both the estimation of the link function and the estimation of the index $\theta_{0}$. Actually, imposing these constraints on the bandwidth is for ease of exposition in the proofs.

Let $\mu_{\theta}(x)=E\left(X \mid \theta^{\top} X=\theta^{\top} x\right), \nu_{\theta}(x)=\mu_{\theta}(x)-x, w_{\theta}(x)=E\left(X X^{\top} \mid \theta^{\top} X=\theta^{\top} x\right), W_{0}(x)=\nu_{\theta_{0}}(x) \nu_{\theta_{0}}(x)$. Let $A^{+}$denote the Moore-Penrose inverse of a symmetric matrix $A$. Recall that $K$ is a symmetric density function. Thus, $\int K(v) d v=1$ and $\int v K(v) d v=0$. For ease of exposition, we further assume that $\mu_{2}=$ $\int v^{2} K(v) d v=1$. Otherwise, we can redefine $K(v):=\mu_{2}^{1 / 2} K\left(\mu_{2}^{1 / 2} v\right)$.

We have the following asymptotic results for the estimators.

Theorem 3.1 (Speed of algorithm) Let $\theta_{\tau}$ be the value calculated in Step 3 after $\tau$ iterations. Suppose assumptions (C1)-(C5) hold. If $h_{\tau} \rightarrow 0$ and $\left|\theta_{\tau}-\theta_{0}\right| / h_{\tau}^{2} \rightarrow 0$, we have

$$
\theta_{\tau+1}-\theta_{0}=\frac{1}{2}\left\{\left(I-\theta_{0} \theta_{0}^{\top}\right)+o(1)\right\}\left(\theta_{\tau}-\theta_{0}\right)+\frac{1}{2 \sqrt{n}} N_{n}+O\left(n^{2 \varsigma} h_{\tau}^{4}\right)
$$

almost surely, where $N_{n}=\left[E\left\{g^{\prime}\left(\theta_{0}^{\top} X\right)^{2} W_{0}(X)\right\}\right]^{+} n^{-1 / 2} \sum_{i=1}^{n} g^{\prime}\left(\theta_{0}^{\top} X_{i}\right) \nu_{\theta_{0}}\left(X_{i}\right) \varepsilon_{i}=O_{p}\left(n^{-1 / 2}\right)$.

Theorem 3.1 indicates that the algorithm converges at a geometric rate, i.e. after each iteration, the estimation error reduces by half approximately. By Theorem 3.1 and the bandwidth requirement in the algorithm, we have

$$
\left|\theta_{\tau+1}-\theta_{0}\right|=\left\{\frac{1}{2}+o(1)\right\}\left|\theta_{\tau+1}-\theta_{0}\right|+O\left(n^{-1 / 2}+n^{2 \varsigma} h_{\tau}^{4}\right)
$$

Starting with $\left|\theta_{1}-\theta_{0}\right|=C n^{-\alpha}$, in order to achieve root- $n$ consistency, say $\left|\theta_{k}-\theta_{0}\right| \leq c n^{-1 / 2}$ i.e. $2^{-k} C n^{-\alpha} \leq$ $c n^{-1 / 2}$, the number of iterations $k$ can be calculated roughly by

$$
k=\left\{\left(\frac{1}{2}-\alpha\right) \log n+\log (C / c)\right\} / \log 2 .
$$

Based on Theorem 3.1, we immediately have the following limiting distribution.

Theorem 3.2 (Efficiency of estimator) Under the conditions (C1)-(C5), we have

$$
\sqrt{n}\left(\hat{\theta}-\theta_{0}\right) \stackrel{\mathcal{L}}{\rightarrow} N\left(0, \Sigma_{0}\right)
$$

where $\Sigma_{0}=\left[E\left\{g^{\prime}\left(\theta_{0}^{\top} X\right)^{2} W_{0}(X)\right\}\right]^{+} E\left\{g^{\prime}\left(\theta_{0}^{\top} X\right)^{2} W_{0}(X) \varepsilon^{2}\right\}\left[E\left\{g^{\prime}\left(\theta_{0}^{\top} X\right)^{2} W_{0}(X)\right\}\right]^{+}$. 
By choosing a similar trimming function, the estimators in Härdle, Hall and Ichimura (1993) and Ichimura (1993) have the same asymptotic covariance matrix as Theorem 3.2. If we further assume that the conditional distribution of $Y$ given $X$ belongs to a canonical exponential family

$$
f_{Y \mid X}(y \mid x)=\exp \{y \eta(x)-\mathcal{B}(\eta(x))+\mathcal{C}(y)\}
$$

for some known functions $\mathcal{B}, \mathcal{C}$ and $\eta$, then $\Sigma_{0}$ is the lower information bound in the semiparametric sense (Bickel, Klaassen, Ritov and Wellner, 1993). See also the proofs in Carroll, Fan, Gijbels and Wand (1997) and Härdle, Hall and Ichimura (1993). In other words, our estimator is the most efficient in the semiparametric sense.

For the estimation of the single-index model, it was generally believed that undersmoothing the link function must be employed in order to allow the estimator of the parameters to achieve root- $n$ consistency. However, Härdle, Hall and Ichimura (1993) established that undersmoothing the link function is not necessary. They derived an asymptotic expansion of the sum of squared residuals. We also derive an asymptotic expansion but of the estimator $\widehat{\theta}$ itself. This allows us to measure the higher order cost of estimating the link function. We use the expansion to propose an automatic bandwidth selection procedure for the index. Let $f_{\theta_{0}}($.$) be the density function of \theta_{0}^{\top} X$.

Theorem 3.3 (Higher Order Expansion) Under conditions (C1)-(C5) and $\varepsilon_{i}$ is independent of $X_{i}$, we have almost surely

$$
\hat{\theta}-\theta_{0}=\mathcal{E}_{n}+\frac{c_{1, n}}{n h}+c_{2, n} h^{4}+\mathcal{H}_{n}+O\left\{n^{2 \varsigma} \gamma_{n}^{3}\right\}
$$

where $\gamma_{n}=h^{2}+(n h / \log n)^{-1 / 2}$,

$$
\mathcal{E}_{n}=\left(W_{n}\right)^{+} \sum_{i=1}^{n} \rho_{n}\left\{f_{\theta_{0}}\left(X_{j}\right)\right\} g^{\prime}\left(\theta_{0}^{\top} X_{i}\right) \nu_{\theta_{0}}\left(\theta_{0}^{\top} X_{i}\right) \varepsilon_{i},
$$

with $W_{n}=n^{-1} \sum_{j=1}^{n} \rho_{n}\left\{f_{\theta_{0}}\left(X_{j}\right)\right\}\left(g^{\prime}\left(\theta_{0}^{\top} X_{i}\right)\right)^{2} \nu_{\theta_{0}}\left(X_{j}\right) \nu_{\theta_{0}}^{\top}\left(X_{j}\right), \mathcal{H}_{n}=O\left\{n^{-1 / 2} \gamma_{n}+n^{-1} h^{-1 / 2}\right\}$ with $E\left\{\mathcal{H}_{n} \mathcal{E}_{n}\right\}$ $=o\left\{(n h)^{-2}+h^{8}\right\}$ and

$$
\begin{gathered}
c_{1, n}=\int K^{2}(v) v^{2} d v \sigma^{2}\left(n W_{n}\right)^{-1} \sum_{j=1}^{n} \rho_{n}\left\{f_{\theta}\left(X_{j}\right)\right\}\left\{\nu_{\theta_{0}}^{\prime}\left(X_{j}\right)+f_{0}^{\prime}\left(X_{j}\right) \nu_{\theta_{0}}\left(X_{j}\right) / f_{\theta_{0}}\left(X_{j}\right)\right\}, \\
c_{2, n}=\frac{1}{4}\left(\int K(v) v^{4} d v-1\right)\left(n W_{n}\right)^{-1} \sum_{j=1}^{n} \rho_{n}\left\{f_{\theta}\left(X_{j}\right)\right\} g^{\prime}\left(\theta_{0}^{\top} X_{j}\right) g^{\prime \prime}\left(\theta_{0}^{\top} X_{j}\right) \nu_{\theta_{0}}^{\prime \prime}\left(X_{j}\right) .
\end{gathered}
$$


Because $K(v)$ is a density function and we constrain that $\int v^{2} K(v)=1$, it follows that $\mu_{4}=\int K(v) v^{4} d v>$ 1. In the expansion of $\hat{\theta}-\theta_{0}$, the first term $\mathcal{E}_{n}$ does not depend on $h$. The second and third terms are the leading term among the remainders. The higher order properties of this estimator are better than those of the AD method, see Nishiyama and Robinson (2000), and indeed do not reflect a curse of dimensionality.

To minimize the stochastic expansion, it is easy to see that the bandwidth should be proportional to $n^{-1 / 5}$. Moreover, by Theorem 3.2 we consider the Mahalanobis distance

$$
\left(\hat{\theta}-\theta_{0}\right)^{\top} \Sigma_{0}^{+}\left(\hat{\theta}-\theta_{0}\right)=T_{n}+o\left\{h^{8}+(n h)^{-2}\right\}
$$

where

$$
T_{n}=\left(\mathcal{E}_{n}+\frac{c_{1, n}}{n h}+c_{2, n} h^{4}+\mathcal{H}_{n}\right)^{\top} \Sigma_{0}^{+}\left(\mathcal{E}_{n}+\frac{c_{1, n}}{n h}+c_{2, n} h^{4}+\mathcal{H}_{n}\right)
$$

is the leading term. We have by Theorem 3.3 that

$$
E T_{n}=E\left(\mathcal{E}_{n}^{\top} \Sigma_{0}^{+} \mathcal{E}_{n}\right)+\left(\frac{c_{1}}{n h}+c_{2} h^{4}\right)^{\top} \Sigma_{0}^{+}\left(\frac{c_{1}}{n h}+c_{2} h^{4}\right)+o\left\{h^{8}+(n h)^{-2}\right\}
$$

where $c_{1}=\int K^{2}(v) v^{2} d v \sigma^{2} W_{0}^{+} E\left\{\nu_{0}^{\prime}(X)+f^{-1}(X) f^{\prime}(X) \nu_{0}(X)\right\}, W_{0}=E\left\{\left(g^{\prime}\left(\theta_{0}^{\top} X\right)\right)^{2} \nu_{\theta_{0}}(X) \nu_{\theta_{0}}^{\top}(X)\right\}$ and

$$
c_{2}=\frac{1}{4}\left(\int K(v) v^{4} d v-1\right) W_{0}^{+} E\left[g^{\prime}\left(\theta_{0}^{\top} X\right) g^{\prime \prime}\left(\theta_{0}^{\top} X\right) \nu_{\theta_{0}}^{\prime \prime}(X)\right] .
$$

Note that $E\left(\mathcal{E}_{n}^{\top} \Sigma_{0}^{+} \mathcal{E}_{n}\right)$ does not depend on $h$. By minimizing $E T_{n}$ with respective to $h$, the optimal bandwidth should be

$$
h_{\theta}=\left\{\frac{\left(9 r_{2}^{2}+16 r_{1}\right)^{1 / 2}-3 r_{2}}{8}\right\}^{1 / 5} n^{-1 / 5}
$$

where $r_{1}=c_{1}^{\top} \Sigma_{0}^{+} c_{1} /\left(c_{2}^{\top} \Sigma_{0}^{+} c_{2}\right)$ and $r_{2}=c_{1}^{\top} \Sigma_{0}^{+} c_{2} / c_{2}^{\top} \Sigma_{0}^{+} c_{2}$. As a comparison, we consider the optimal bandwidth for the estimation of the link function $g$. By Lemma 5.1 and Theorem 3.2 , if $f_{\theta_{0}}(v)>0$ we have

$$
\hat{g}(v)=g(v)+\frac{1}{2} g^{\prime \prime}(v)^{2} h^{2}+\frac{1}{n f_{\theta_{0}}(v)} \sum_{i=1}^{n} K_{h}\left(\theta_{0}^{\top} X_{i}-v\right) \varepsilon_{i}+O_{P}\left(n^{-1 / 2}+h^{2} \gamma_{n}\right) .
$$

In other words, the link function can be estimated with the efficiency as if the index parameter vector is known. A brief proof for (6) is given in section 5. It follows that

$$
|\hat{g}(v)-g(v)|^{2}=S_{n}(v)+O_{P}\left\{\left(n^{-1 / 2}+h^{2} \gamma_{n}\right) \gamma_{n}\right\}
$$

where the leading term is $S_{n}(v)=\left[\frac{1}{2} g^{\prime \prime}(v)^{2}+\left\{n f_{\theta_{0}}(v)\right\}^{-1} \sum_{i=1}^{n} K_{h}\left(\theta_{0}^{\top} X_{i}-v\right) \varepsilon_{i}\right]^{2}$. Suppose we are interested in constant bandwidth in region $[a, b]$ with weight $w(v)$. Minimizing $\int_{[a, b]} E S_{n}(v) w(v) d v$ with respect to $h$, 
we have the optimal bandwidth for the estimation of the link function is

$$
h_{g}=\left[\frac{\int K^{2}(v) d v \int_{[a, b]} f_{\theta_{0}}^{-1}(v) \sigma_{\theta_{0}}^{2}(v) w(v) d v}{\int_{[a, b]} g^{\prime \prime}(v)^{2} w(v) d v}\right]^{1 / 5} n^{-1 / 5} .
$$

It is noticeable that the optimal bandwidth for the estimation of the parameter vector $\theta_{0}$ is of the same order as that for the estimation of the link function. In other words, under-smoothing may lose efficiency for the estimation of $\theta_{0}$ in the higher order sense. These optimal bandwidth $h_{\theta}^{o p t}$ and $h_{g}^{o p t}$ can be consistently estimated by plug-in methods; see Ruppert et al (1995).

Although the optimal bandwidth for the estimation of $\theta$ is different from that for the link function, its estimation such as the plug-in method may be very unstable because of the estimation of second order derivatives. Moreover, its estimation needs another pilot parameter which is again hard to choose. In

practice it is convenient to apply $h_{g}^{o p t}$ for $h_{\theta}^{o p t}$ directly, and since $h_{g}^{o p t}$ and $h_{\theta}^{o p t}$ have the same order, the loss of efficiency in doing so should be small. For the former, there are a number of estimation methods such as $\mathrm{CV}$ and GCV methods. If CV methods is used, in each iteration with the latest estimator $\theta$, the bandwidth is selected by minimizing

$$
\hat{h}_{g}=\underset{h}{\operatorname{argmin}} n^{-1} \sum_{j=1}^{n}\left\{Y_{j}-\hat{g}_{j}^{\theta}\left(\theta^{\top} X_{j}\right)\right\}^{2}
$$

where $\hat{g}_{j}^{\theta}(v)$ is the delete-one-observation estimator of the link function, i.e. the estimator of $\hat{g}^{\theta}(v)$ in (3) using data $\left\{\left(X_{i}, Y_{i}\right), i \neq j\right\}$. Another advantage for this approach is that we can also obtain the estimator for the link function.

\section{Numerical Results}

In the following calculation, the Gaussian kernel function and the trimming function (44) with $\varsigma=1 / 20$ and $c_{0}=0.01$ are used. A MATLAB code rMAVE.m for the calculations below is available at

$$
\text { http://www.stat.nus.edu.sg/\% YEstaxyc }
$$

In the first example, we check the behavior of bandwidths $h_{g}$ and $h_{\theta}$. We consider two sets of simulations to investigate the finite performance of our estimation method, and to compare the bandwidths for the estimation of the link function $g$ and the single-index $\theta_{0}$. Our models are

model A: $y=\left(\theta_{0}^{\top} X\right)^{2}+0.2 \varepsilon, \quad$ model B: $y=\cos \left(\theta_{0}^{\top} X\right)+0.2 \varepsilon$, 
where $\theta_{0}=(3,2,2,1,0,0,-1,-2,-2,-3)^{\top} / 6, X \sim N_{10}(0, I)$, and $\varepsilon \sim N(0,1)$ is independent of $X$. The ADE method was used to choose the initial value of $\theta$. With different sample size $n$ and bandwidth $h$, we estimate the model and calculate estimation errors

$$
\operatorname{err}_{\theta}=\left\{1-\left|\theta_{0}^{\top} \hat{\theta}\right|\right\}^{1 / 2}, \quad \operatorname{err}_{g}=\frac{1}{n} \sum_{j=1}^{n} \rho_{n}\left\{\hat{f}_{\hat{\theta}}\left(\hat{\theta}^{\top} X_{j}\right)\right\}\left|\hat{g}^{\hat{\theta}}\left(\hat{\theta}^{\top} X_{j}\right)-g\left(\theta_{0}^{\top} X_{j}\right)\right|,
$$

where $\hat{g}^{\hat{\theta}}\left(\hat{\theta}^{\top} X_{j}\right)$ is defined in (3). With 200 replications, we calculate the mean errors mean $\left(e r r_{\theta}\right)$ and mean $\left(\operatorname{err}_{g}\right)$. The results are shown in Figure 1.

We have the following observations. (1) Notice that $n^{1 / 2}$ mean $\left(\operatorname{err}_{\theta}\right)$ tends to decrease as $n$ increases, which means the estimation error $\operatorname{err}_{\theta}$ enjoys a root- $n$ consistency (and slightly faster for finite sample size). (2) Notice that the U-shape curves of err $_{\theta}$ has a wider bottom than those of err $_{g}$. Thus, the estimation of $\theta_{0}$ is more robust to the bandwidth than the estimation of $g$. (3) Let $h_{\theta}^{\text {opt }}=\arg \min _{h} \operatorname{mean}_{\left(\text {err }_{\theta}\right)}$ and $h_{g}^{o p t}=\arg \min _{h}$ mean $\left(e r r_{g}\right)$. Then $h_{\theta}^{o p t}$ and $h_{g}^{o p t}$ represent the best bandwidths respectively for the estimation of the link function $g$ and the single-index $\theta_{0}$. Notice that $h_{\theta}^{o p t} / h_{g}^{o p t}$ tends to increase as $n$ increases, which means the optimal bandwidth for the estimation of $\theta_{0}$ tends to zero not faster than that for the estimation of link function. Thus the under-smoothing bandwidth is not optimal.

Next, we compare our method with some of the existing estimation methods including ADE in Härdle and Stocker (1993), MAVE, the method in Hristache et al (2001), called HJS hereafter, the SIR and pHd methods in Li $(1991,1992)$ and SLS in Ichimura (1993). For SLS, we use the algorithm in Friedman (1984) in the calculation. The algorithm has best performance among those proposed for the minimization of SLS, such as Weisberg and Welsh (1994) and Fan and Yao (2003). We consider the following model used in Hristache et al (2001),

$$
Y=\left(\theta_{0}^{\top} X\right)^{2} \exp \left(a \theta_{0}^{\top} X\right)+\sigma \varepsilon
$$

where $X=\left(\mathbf{x}_{1}, \cdots, \mathbf{x}_{10}\right)^{\top}, \theta_{0}=(1,2,0, \ldots, 0)^{\top} / \sqrt{5}, \mathbf{x}_{1}, \cdots, \mathbf{x}_{10}, \varepsilon$ are independent and $\varepsilon \sim N(0,1)$. For the covariates $X:\left(\mathbf{x}_{k}+1\right) / 2 \sim \operatorname{Beta}(\tau, 1)$ for $k=1, \cdots, p$. Parameter $a$ is introduced to control the shape of function. If $a=0$, the structure is symmetric; the bigger it is, the more monotonic the function is.

Following Hristache et al (2001), we use the absolute deviation $\sum_{j=1}^{p}\left|\hat{\theta}_{j}-\theta_{j}\right|$ to measure the estimation errors. The calculation results for different $\sigma$ and $\tau$ based on 250 replications are shown in Table 1 . We have the following observations from Table 1. Our methods has much better performance than ADE and the method of Hristache et al (2001). For each simulation, the better one of SIR and pHd is reported in Table 
Figure 1: The wide solid lines are the values of $\log \left\{n^{1 / 2}\right.$ mean $\left.\left(\operatorname{err}_{\theta}\right)\right\}$ and the narrow lines are the values of $\log \left\{n^{1 / 2}\right.$ mean $\left.\left(\operatorname{err}_{g}\right)\right\}$ (re-scaled for easier visualisation). The dotted vertical lines correspond to the bandwidths $h_{\theta}$ and $h_{g}$ respectively.
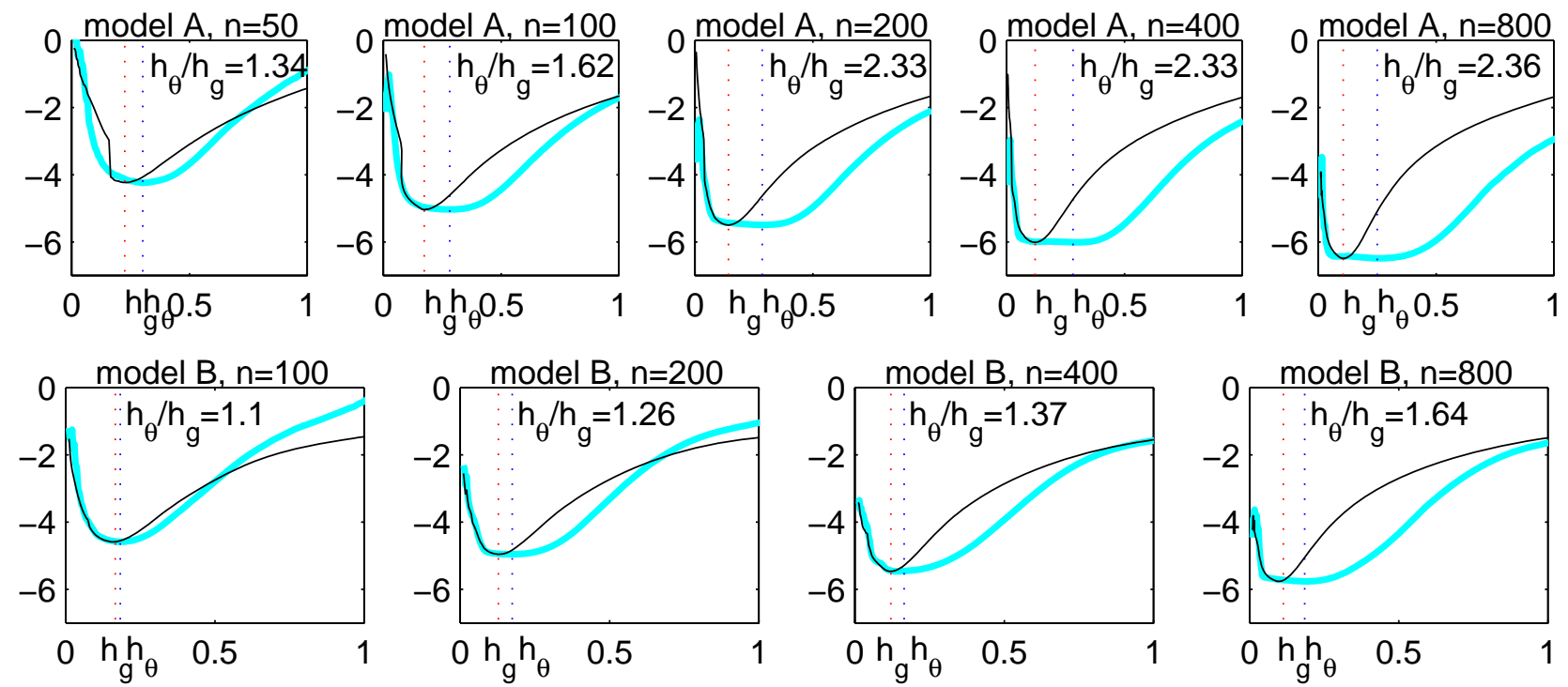

Table 1. Average estimation errors $\sum_{j=1}^{p}\left|\hat{\theta}_{j}-\theta_{j}\right|$ and their standard deviations (in square bracket) for model (7).

\begin{tabular}{|c|c|c|c|c|c|c|c|c|c|c|}
\hline \multirow[b]{2}{*}{$\mathrm{n}$} & \multirow[b]{2}{*}{$\sigma$} & \multirow[b]{2}{*}{$\tau$} & \multicolumn{5}{|c|}{$a=1$} & \multicolumn{3}{|c|}{$a=0$} \\
\hline & & & $\mathrm{ADE}^{*}$ & HJS* & $\mathrm{SIR} / \mathrm{pHd}$ & SLS & MAVE & $\mathrm{SIR} / \mathrm{pHd}$ & SLS & MAVE \\
\hline \multirow[t]{2}{*}{200} & 0.1 & 1 & 0.6094 & 0.1397 & 0.6521 & 0.0645 & 0.0514 & 0.7500 & 0.6910 & 0.0936 \\
\hline & & & & & [0.1569] & {$[0.0258]$} & [0.0152] & {$[0.1524]$} & [1.2491] & {$[0.0255]$} \\
\hline \multirow[t]{2}{*}{200} & 0.2 & 1 & 0.6729 & 0.2773 & 0.6976 & 0.1070 & 0.0934 & 0.7833 & 0.8937 & 0.1809 \\
\hline & & & & & [0.1759] & {$[0.0375]$} & [0.0294] & {$[0.1666]$} & [1.3192] & {$[0.0483]$} \\
\hline \multirow[t]{2}{*}{400} & 0.1 & 0.75 & 0.7670 & 0.1447 & 0.3778 & 0.1151 & 0.0701 & 0.6037 & 0.0742 & 0.0562 \\
\hline & & & & & [0.0835] & {$[0.0410]$} & [0.0197] & {$[0.1134]$} & [0.0193] & {$[0.0146]$} \\
\hline \multirow[t]{2}{*}{400} & 0.1 & 1 & 0.4186 & 0.0822 & 0.4868 & 0.0384 & 0.0295 & 0.5820 & 0.5056 & 0.0613 \\
\hline & & & & & [0.1149] & {$[0.0125]$} & [0.0096] & {$[0.1084]$} & [1.0831] & {$[0.0167]$} \\
\hline \multirow[t]{2}{*}{400} & 0.1 & 1.5 & 0.2482 & 0.0412 & 0.5670 & 0.0208 & 0.0197 & 0.5760 & 0.0923 & 0.0669 \\
\hline & & & & & {$[0.1524]$} & {$[0.0063]$} & [0.0056] & {$[0.1215]$} & {$[0.0257]$} & {$[0.0175]$} \\
\hline \multirow[t]{2}{*}{400} & 0.2 & 1 & 0.4665 & 0.1659 & 0.5249 & 0.0654 & 0.0607 & 0.6084 & 0.7467 & 0.1229 \\
\hline & & & & & [0.1353] & {$[0.0207]$} & [0.0178] & [0.1064] & [1.2655] & {$[0.0357]$} \\
\hline \multirow[t]{2}{*}{400} & 0.4 & 1 & 0.5016 & 0.3287 & 0.6328 & 0.1262 & 0.1120 & 0.6994 & 0.9977 & 0.2648 \\
\hline & & & & & {$[0.1386]$} & {$[0.0406]$} & [0.0339] & [0.1370] & [1.2991] & [0.1880] \\
\hline
\end{tabular}

\footnotetext{
* The values are adopted from Hristache et al (2001)
} 
1 , suggesting that these methods are not so competitive. Actually the main application of SIR and pHd is not in the estimation of single-index models. See Li (1991, 1992). For SLS, its performance depends much on the data and the model. If the model is easy to estimate (such as monotone and having big signal/noise ratio), it performance quite well. But overall SLS is still not so good as MAVE. The proposed method has the best performance in all the simulations we have done.

\section{$5 \quad$ Proof of Theorems}

Let $f_{\theta}(v)$ be the density function of $\theta^{\top} X$ and $\Lambda_{n}=\left\{x:|x|<n^{c}, f_{\theta}(x)>n^{-2 \varsigma}, \theta \in \Theta_{n}\right\}$ where $c>1 / 3$ and $\varsigma>0$ is defined in (C5). Suppose $A_{n}$ is a random matrix depending on $x$ and $\theta$. By $A_{n}=\mathcal{O}\left(a_{n}\right)$ (or $A_{n}=\mathcal{O}\left(a_{n}\right)$ ) we mean that all elements in $A_{n}$ are $O_{a . s .}\left(a_{n}\right)$ (or $o_{a . s .}\left(a_{n}\right)$ ) uniformly for $\theta \in \Theta_{n}$ and $x \in \Lambda_{n}$. Let $\delta_{n}=(n h / \log n)^{-1 / 2}, \gamma_{n}=h^{2}+\delta_{n}$ and $\delta_{\theta}=\left|\theta-\theta_{0}\right|$. For any vector $V(v)$ of functions of $v$, we define $(V(v))^{\prime}=d V(v) / d v$.

Suppose $\left(X_{i}, Z_{i}\right), i=1,2, \ldots, n$, are i.i.d. samples from $(X, Z)$. Let $X_{i x}=X_{i}-x$,

$$
\begin{gathered}
s_{k}^{\theta}(x)=n^{-1} \sum_{i=1}^{n} K_{h}\left(\theta^{\top} X_{i x}\right)\left\{\theta^{\top} X_{i x} / h\right\}^{k}, \quad t_{k}^{\theta}(x)=n^{-1} \sum_{i=1}^{n} K_{h}\left(\theta^{\top} X_{i x}\right)\left\{\theta^{\top} X_{i x} / h\right\}^{k} X_{i}, \\
w_{k}^{\theta}(x)=n^{-1} \sum_{i=1}^{n} K_{h}\left(\theta^{\top} X_{i x}\right)\left\{\theta^{\top} X_{i x} / h\right\}^{k} X_{i} X_{i}^{\top}, \quad e_{k}^{\theta}(x)=n^{-1} \sum_{i=1}^{n} K_{h}\left(\theta^{\top} X_{i x}\right)\left\{\theta^{\top} X_{i x} / h\right\}^{k} \varepsilon_{i}, \\
\epsilon_{k}^{\theta}=s_{k}^{\theta}(x)-E s_{k}^{\theta}(x), \xi_{k}^{\theta}=t_{k}^{\theta}(x)-E t_{k}^{\theta}(x), D_{n, k}^{\theta}(x)=s_{2}^{\theta}(x) s_{k}^{\theta}(x)-s_{1}^{\theta}(x) s_{k+1}^{\theta}(x), E_{n, k}^{\theta}=s_{0}^{\theta}(x) s_{k+1}^{\theta}(x)- \\
s_{1}^{\theta}(x) s_{k}^{\theta}(x) \text { for } k=1,2, \ldots \text { For any random variable } Z \text { and its random observations } Z_{i}, i=1, \ldots, n, \text { let } \\
T_{n, k}^{\theta}(Z \mid x)=s_{2}^{\theta}(x) n^{-1} \sum_{i=1}^{n} K_{h}^{\theta}\left(X_{i x}\right)\left(\theta^{\top} X_{i x} / h\right)^{k} Z_{i}-s_{1}^{\theta}(x) n^{-1} \sum_{i=1}^{n} K_{h}^{\theta}\left(X_{i x}\right)\left(\theta^{\top} X_{i x} / h\right)^{k+1} Z_{i}, \\
S_{n, k}^{\theta}(Z \mid x)=s_{0}^{\theta}(x) n^{-1} \sum_{i=1}^{n} K_{h}^{\theta}\left(X_{i x}\right)\left(\theta^{\top} X_{i x} / h\right)^{k+1} Z_{i}-s_{1}^{\theta}(x) n^{-1} \sum_{i=1}^{n} K_{h}^{\theta}\left(X_{i x}\right)\left(\theta^{\top} X_{i x} / h\right)^{k} Z_{i} .
\end{gathered}
$$

By the Taylor expansion of $g\left(\theta_{0}^{\top} X_{i}\right)$ at $\theta_{0}^{\top} x$, we have

$$
\begin{aligned}
g\left(\theta_{0}^{\top} X_{i}\right) & =g\left(\theta_{0}^{\top} x\right)+\sum_{k=1}^{5} \frac{1}{k !} g^{(k)}\left(\theta_{0}^{\top} x\right)\left\{\theta^{\top} X_{i x}+\left(\theta_{0}-\theta\right)^{\top} X_{i x}\right\}^{k}+O\left(\left\{\theta^{\top} X_{i x}+\left(\theta_{0}-\theta\right)^{\top} X_{i x}\right\}^{6}\right) \\
& =g\left(\theta_{0}^{\top} x\right)+A^{\theta}\left(x, X_{i}\right)+B^{\theta}\left(x, X_{i}\right)\left(\theta_{0}-\theta\right)+O\left\{\left(\theta^{\top} X_{i x}\right)^{6}+\delta_{\theta}^{3}\left(\left|X_{i}\right|^{6}+|x|^{6}\right)\right\},
\end{aligned}
$$

where $A^{\theta}\left(x, X_{i}\right)=\sum_{\ell=1}^{5}(k !)^{-1} g^{(k)}\left(\theta_{0}^{\top} x\right)\left(\theta^{\top} X_{i x}\right)^{k}$ and

$$
B^{\theta}\left(x, X_{i}\right)=\sum_{k=1}^{5} \frac{1}{(k-1) !} g^{(k)}\left(\theta_{0}^{\top} x\right)\left(\theta^{\top} X_{i x}\right)^{k-1} X_{i x}^{\top}+\frac{1}{2} g^{\prime \prime}\left(\theta_{0}^{\top} x\right)\left(\theta-\theta_{0}\right)^{\top} X_{i x} X_{i x}^{\top} .
$$


For ease of exposition, we simplify the notation and abbreviate $g$ for $g\left(\theta_{0}^{\top} x\right)$ and $g^{\prime}, g^{\prime \prime}, g^{\prime \prime \prime}$ for $g^{\prime}\left(\theta_{0}^{\top} x\right)$, $g^{\prime \prime}\left(\theta_{0}^{\top} x\right), g^{\prime \prime \prime}\left(\theta_{0}^{\top} x\right)$ respectively. Without causing confusion, we write $f_{\theta}\left(\theta^{\top} x\right)$ as $f_{\theta}, f_{\theta}\left(\theta^{\top} X_{j}\right)$ as $f_{\theta}\left(X_{j}\right)$ and $K_{h}\left(\theta^{\top} X_{i j}\right)$ as $K_{h}^{\theta}\left(X_{i j}\right)$. Similar notations are used for the other functions.

\section{Lemma 5.1 (Link function) Let}

$$
\Sigma_{n}^{\theta}(x)=n^{-1} \sum_{i=1}^{n} K_{h}^{\theta}\left(X_{i x}\right)\left(\begin{array}{c}
1 \\
\theta^{\top} X_{i x} / h
\end{array}\right)\left(\begin{array}{c}
1 \\
\theta^{\top} X_{i x} / h
\end{array}\right)^{\top}
$$

and

$$
\left(\begin{array}{c}
a_{\theta}(x) \\
d_{\theta}(x) h
\end{array}\right)=\left\{n \Sigma_{n}^{\theta}(x)\right\}^{-1} \sum_{i=1}^{n} K_{h}^{\theta}\left(X_{i x}\right)\left(\begin{array}{c}
1 \\
\theta^{\top} X_{i x} / h
\end{array}\right) Y_{i} .
$$

Under assumptions (C2)-(C5), we have

$$
\begin{gathered}
a_{\theta}(x)=g\left(\theta_{0}^{\top} x\right)+A_{n}^{\theta}(x) h^{2}+B_{n}^{\theta}(x)\left(\theta_{0}-\theta\right)+V_{n}^{\theta}(x)+\mathcal{O}\left(h^{2} \gamma_{n}^{2}+\delta_{\theta}^{3}\right)\left(1+|x|^{6}\right), \\
d_{\theta}(x) h=g^{\prime}\left(\theta_{0}^{\top} x\right) h+\tilde{A}_{n}^{\theta}(x) h^{2}+\tilde{B}_{n}^{\theta}(x)\left(\theta_{0}-\theta\right) h+\tilde{V}_{n}^{\theta}(x)+\mathcal{O}\left(h^{2} \gamma_{n}^{2}+\delta_{\theta}^{3}\right)\left(1+|x|^{6}\right),
\end{gathered}
$$

where

$$
\begin{aligned}
& A_{n}^{\theta}(x)=\frac{1}{2} g^{\prime \prime}+\frac{1}{4}\left\{\left(\mu_{4}-1\right) g^{\prime \prime} f_{\theta}^{-2}\left(f_{\theta} f_{\theta}^{\prime \prime}-2\left(f_{\theta}^{\prime}\right)^{2}\right)+\frac{1}{24} \mu_{4} g^{(4)}\right\} h^{2}+H_{1, n}^{\theta}(x), \\
& \tilde{A}_{n}^{\theta}(x)=\frac{1}{2} g^{\prime \prime}\left(\mu_{4}-1\right) f_{\theta}^{-1} f_{\theta}^{\prime} h+\frac{1}{6} g^{(3)} \mu_{4} h+\frac{1}{2} g^{\prime \prime} f_{\theta}^{-1}\left(\epsilon_{3}^{\theta}-\epsilon_{1}^{\theta}\right)+\mathcal{O}\left(h \gamma_{n}\right), \\
& B_{n}^{\theta}(x)=g^{\prime} \nu_{\theta}+\mathcal{O}\left(\gamma_{n}+\delta_{\theta}\right), \quad \tilde{B}_{n}^{\theta}(x)=g^{\prime}\left(\theta_{0}^{\top} x\right) f_{\theta}^{-1}\left\{f_{\theta} \nu_{\theta}(x)\right\}^{\prime}+\mathcal{O}\left(\gamma_{n}\right),
\end{aligned}
$$

where $H_{1, n}^{\theta}(x)=\frac{1}{2} g^{\prime \prime}\left(\theta_{0}^{\top} x\right)\left\{f_{\theta}^{-1}\left(\epsilon_{2}^{\theta}-\epsilon_{0}^{\theta}\right)+\left(2-\mu_{4}\right) f_{\theta}^{-2} f_{\theta}^{\prime} h \epsilon_{1}^{\theta}-f_{\theta}^{-2} f_{\theta}^{\prime} h \epsilon_{3}^{\theta}\right\}+\frac{1}{6} f_{\theta}^{-1} g^{\prime \prime \prime} h \epsilon_{3}^{\theta}$ and $V_{n}^{\theta}(x)=f_{\theta}^{-1} e_{0}^{\theta}-$ $f_{\theta}^{-2} f_{\theta}^{\prime} h e_{1}^{\theta}+\mu_{4} f_{\theta}^{-2} f_{\theta}^{\prime \prime} h^{2} e_{0}^{\theta} / 2+f_{\theta}^{-2}\left(e_{0}^{\theta} \epsilon_{2}^{\theta}-e_{1}^{\theta} \epsilon_{1}^{\theta}\right)-\mu_{4} f_{\theta}^{-2} f_{\theta}^{\prime \prime \prime} h^{3} e_{1}^{\theta}+\left\{f_{\theta}^{-2}\left(f_{\theta}^{\prime}\right)^{2}-\left(\mu_{4}+1\right) f_{\theta}^{-1} f_{\theta}^{\prime \prime}\right\}\left\{f_{\theta}^{-1} h^{2} e_{0}^{\theta}-\right.$ $\left.f_{\theta}^{-2} f_{\theta}^{\prime} h^{3} e_{1}^{\theta}\right\}-f_{\theta}^{-1}\left(\epsilon_{0}^{\theta}+\epsilon_{1}^{\theta}\right)\left\{f_{\theta}^{-1} e_{0}^{\theta}-f_{\theta}^{-2} f_{\theta}^{\prime} e_{1}^{\theta}\right\}+2 f_{\theta}^{-2} f_{\theta}^{\prime} h \epsilon_{1}^{\theta} f_{\theta}^{-1} e_{0}^{\theta}$ and $\tilde{V}_{n}^{\theta}(x)=f_{\theta}^{-1} e_{1}^{\theta}+f_{\theta}^{-2} f_{\theta}^{\prime \prime} h^{2} e_{1}^{\theta} / 2+f_{\theta}^{-2}\left(\epsilon_{0}^{\theta} e_{1}^{\theta}-\right.$ $\left.\epsilon_{1}^{\theta} e_{0}^{\theta}\right)-f_{\theta}^{-2} f_{\theta}^{\prime} h e_{0}^{\theta}+f_{\theta}^{-1} \epsilon_{0}^{\theta}\left[-\left(\mu_{4}+1\right) f_{\theta}^{-1} f_{\theta}^{\prime \prime} h^{2} / 2-f_{\theta}^{-1}\left(\epsilon_{0}^{\theta}+\epsilon_{1}^{\theta}\right)+f_{\theta}^{-2}\left(f_{\theta}^{\prime}\right)^{2} h^{2}\right]$.

Lemma 5.2 (Summations) Let $\eta_{n}^{\theta}(x)=n^{-1} \sum_{i=1}^{n} K_{h}^{\theta}\left(X_{i x}\right) X_{i x} \varepsilon_{i}$. Under conditions (C1)-(C5), we have

$$
\begin{gathered}
\mathcal{A}_{n}^{\theta} \stackrel{\text { def }}{=} n^{-1} \sum_{j=1}^{n} \rho_{n}\left\{s_{0}^{\theta}\left(x_{j}\right)\right\} g^{\prime}\left(\theta_{0}^{\top} X_{j}\right) \eta_{n}^{\theta}\left(X_{j}\right) / s_{0}^{\theta}\left(X_{j}\right)=\mathcal{E}_{n}^{\theta}+r_{n, 0}^{\theta}\left(\theta-\theta_{0}\right)+Q_{n}^{\theta}+\mathcal{O}\left(n^{2 \varsigma} \gamma_{n}^{3}\right), \\
\mathcal{B}_{n}^{\theta} \stackrel{\text { def }}{=}(n h)^{-1} \sum_{j=1}^{n} \rho_{n}\left\{s_{0}^{\theta}\left(X_{j}\right)\right\} e_{k}^{\theta}\left(X_{j}\right) \eta_{n}^{\theta}\left(X_{j}\right) / s_{0}^{\theta}\left(X_{j}\right)=\frac{\tilde{c}_{k, n}}{n h}+R_{n}^{\theta}+\mathcal{O}\left(n^{2 \varsigma} \gamma_{n}^{3}\right), \\
\mathcal{C}_{n}^{\theta} \stackrel{\text { def }}{=} n^{-1} \sum_{j=1}^{n} \rho_{n}\left(s_{0}^{\theta}\left(X_{j}\right)\right) \epsilon_{k}^{\theta}\left(X_{j}\right) \eta_{n}^{\theta}\left(X_{j}\right) / s_{0}^{\theta}\left(X_{j}\right)=M_{n}^{\theta}+\mathcal{O}\left(n^{2 \varsigma} \gamma_{n}^{3}\right),
\end{gathered}
$$


where $\mathcal{E}_{n}^{\theta}=\sum_{i=1}^{n} \rho_{n}\left\{f_{\theta}\left(X_{j}\right)\right\} g^{\prime}\left(\theta^{\top} X_{i}\right) \nu_{\theta}\left(\theta^{\top} X_{i}\right) \varepsilon_{i}, r_{n, 0}^{\theta}=\mathcal{O}(1)$,

$$
\mathcal{E}_{n}^{\theta}=\mathcal{O}\left\{(n / \log n)^{-1 / 2}\right\}, \quad Q_{n}^{\theta}=\mathcal{O}\left\{(n / \log n)^{-1 / 2} \gamma_{n}\right\}, \quad R_{n}^{\theta}=\mathcal{O}\left\{n^{-1 / 2} \delta_{n}\right\}, \quad M_{n}^{\theta}=\mathcal{O}\left\{n^{-1 / 2} \delta_{n}\right\}
$$

with $E\left\{\mathcal{E}_{n}^{\theta} Q_{n}^{\theta}\right\}=o\left(h^{8}+(n h)^{-2}\right), E\left\{\mathcal{E}_{n}^{\theta} R_{n}^{\theta}\right\}=o\left(h^{8}+(n h)^{-2}\right), E\left\{\mathcal{E}_{n}^{\theta} M_{n}^{\theta}\right\}=o\left(h^{8}+(n h)^{-2}\right)$, and $\tilde{c}_{k, n}=$ $\int v^{k+1} K^{2}(v) d v E\left[\rho_{n}\left(f_{\theta}\left(X_{j}\right)\right) f_{\theta}^{-1}\left(X_{j}\right)\left(\nu_{\theta}\left(X_{j}\right) f_{\theta}\left(X_{j}\right)\right)^{\prime}\left(X_{j}\right)\right]$ if $k$ is odd, 0 otherwise.

Lemma 5.3 (Denominator) Let $\mathcal{D}_{n}^{\theta}=n^{-2} \sum_{i, j=1}^{n} \rho_{n}\left(s_{0}^{\theta}\left(X_{j}\right)\right) d_{\theta}^{2}\left(X_{j}\right) K_{h}^{\theta}\left(X_{i j}\right) X_{i j} X_{i j}^{\top} / s_{0}^{\theta}\left(X_{j}\right)$ in the algorithm. Suppose $(\theta, B): p \times p$ is an orthogonal matrix. Then under (C1)-(C5), we have almost surely

$$
\left(\mathcal{D}_{n}^{\theta}\right)^{-1}=\theta \theta^{\top} d_{11}^{\theta} h^{-2}-\theta d_{12}^{\theta} B^{\top} h^{-1}-B\left(d_{12}^{\theta}\right)^{\top} \theta^{\top} h^{-1}+B d_{22}^{\theta} B^{\top},
$$

where

$$
d_{11}^{\theta}=\left(G_{n}^{\theta}\right)^{-1}+\mathcal{O}(1), \quad d_{12}^{\theta}=H_{n}^{\theta} h+\mathcal{O}\left(\gamma_{n}\right), \quad d_{22}^{\theta}=\frac{1}{2}\left(B^{\top} W_{n}^{\theta} B\right)^{-1}+\mathcal{O}\left(\gamma_{n}\right),
$$

with $G_{n}^{\theta}=n^{-1} \sum_{j=1}^{n} \rho_{n}\left(f_{\theta}\left(X_{j}\right)\right) f_{\theta}^{-1}\left(X_{j}\right)\left(g^{\prime}\left(\theta_{0} X_{j}\right)\right)^{2}$ and $H_{n}^{\theta}=\frac{1}{2} n^{-1} \sum_{j=1}^{n} \rho_{n}\left(f_{\theta}\left(X_{j}\right)\right) f_{\theta}^{-1}\left(X_{j}\right)\left\{\left(f_{\theta} \nu_{\theta}\right)^{\prime}\left(X_{j}\right)\right\}^{\top}$ $\left(G_{n}^{\theta}\right)^{-1}\left(g^{\prime}\left(\theta_{0}^{\top} X_{j}\right)\right)^{2} B\left(B^{\top} W_{n}^{\theta} B\right)^{-1}$ and $W_{n}^{\theta}=n^{-1} \sum_{j=1}^{n} \rho_{n}\left\{f_{\theta}\left(X_{j}\right)\right\}\left(g^{\prime}\left(\theta^{\top} X_{i}\right)\right)^{2} \nu_{\theta}\left(X_{j}\right) \nu_{\theta}^{\top}\left(X_{j}\right)$.

Proof of Lemma 5.3 Let $(\theta, B)$ be an orthogonal matrix. It is easy to see that

$$
\begin{gathered}
n^{-1} \sum_{i=1}^{n} K_{h}^{\theta}\left(X_{i x}\right) \theta^{\top} X_{i x} X_{i x}^{\top} \theta=s_{2}^{\theta}(x) h^{2}, \quad n^{-1} \sum_{i=1}^{n} K_{h}^{\theta}\left(X_{i x}\right) \theta^{\top} X_{i x} X_{i x}^{\top} B=\left\{t_{1}^{\theta}(x)-s_{1}^{\theta}(x) x\right\}^{\top} B h, \\
n^{-1} \sum_{i=1}^{n} K_{h}^{\theta}\left(X_{i x}\right) B^{\top} X_{i x} X_{i x}^{\top} B=B^{\top}\left\{w_{0}^{\theta}(x)-t_{0}^{\theta}(x) x^{\top}-x\left(t_{0}^{\theta}(x)\right)^{\top}+x x^{\top} s_{0}^{\theta}(x)\right\} B .
\end{gathered}
$$

Thus

$$
\left(\mathcal{D}_{n}^{\theta}\right)^{-1}=(\theta, B)\left(\begin{array}{cc}
D_{11}^{\theta} h^{2} & \left(D_{12}^{\theta}\right)^{\top} B h \\
B^{\top} D_{12}^{\theta} h & B^{\top} D_{22}^{\theta} B
\end{array}\right)^{-1}(\theta, B)^{\top},
$$

where

$$
\begin{gathered}
D_{11}^{\theta}=n^{-1} \sum_{j=1}^{n} \rho_{n}\left(s_{0}^{\theta}\left(X_{j}\right)\right)\left\{d^{\theta}\left(X_{j}\right)\right\}^{2} s_{2}^{\theta}\left(X_{j}\right) / s_{0}^{\theta}\left(X_{j}\right), \\
D_{12}^{\theta}=n^{-1} \sum_{j=1}^{n} \rho_{n}\left(s_{0}^{\theta}\left(X_{j}\right)\right)\left\{d_{\theta}\left(X_{j}\right)\right\}^{2}\left\{t_{1}^{\theta}\left(X_{j}\right)-s_{1}^{\theta}\left(X_{j}\right) X_{j}\right\}^{\top} / s_{0}^{\theta}\left(X_{j}\right), \\
D_{22}^{\theta}=n^{-1} \sum_{j=1}^{n} \rho_{n}\left(s_{0}^{\theta}\left(X_{j}\right)\right)\left(d_{\theta}\left(X_{j}\right)\right)^{2}\left\{w_{0}^{\theta}\left(X_{j}\right)-t_{0}^{\theta}\left(X_{j}\right) X_{j}^{\top}-X_{j} t_{0}^{\theta}\left(X_{j}\right)+X_{j} X_{j}^{\top} s_{0}^{\theta}\left(X_{j}\right)\right\} / s_{0}^{\theta}\left(X_{j}\right) .
\end{gathered}
$$


By the matrix inversion formula in blocks (Schott, 1997), we have the equation in Lemma 5.3 with $d_{11}=\left\{D_{11}^{\theta}-\left(D_{12}^{\theta}\right)^{\top} B B^{\top}\left(D_{22}^{\theta}\right)^{-1} B B^{\top} D_{12}^{\theta}\right\}^{-1}, d_{12}=d_{11}^{\theta}\left(D_{12}^{\theta}\right)^{\top} B\left(B^{\top} D_{22}^{\theta} B\right)^{-1}, d_{22}^{\theta}=\left\{B^{\top} D_{22}^{\theta} B\right\}^{-1}+$ $d_{11}\left\{B^{\top} D_{22}^{\theta} B\right\}^{-1} B^{\top} D_{12}^{\theta}\left(D_{12}^{\theta}\right)^{\top} B\left\{B^{\top} D_{22}^{\theta} B\right\}^{-1}$. By Lemma 6.1, we have

$$
D_{11}^{\theta}=G_{n}^{-1}+\mathcal{O}(1), \quad D_{12}^{\theta}=H_{n} h+\mathcal{O}\left(\gamma_{n}\right), \quad D_{22}^{\theta}=2 W_{n}+\mathcal{O}\left(\gamma_{n}\right)
$$

Thus, Lemma 5.3 follows.

Lemma 5.4 (Numerator) Let $\mathcal{N}_{n}^{\theta}=n^{-2} \sum_{i, j=1}^{n} \rho_{n}\left(s_{0}^{\theta}\left(X_{j}\right)\right) K_{h}^{\theta}\left(X_{i j}\right) X_{i j}\left\{Y_{i}-a_{\theta}\left(X_{j}\right)-d_{\theta}\left(X_{j}\right) \theta_{0}^{\top} X_{i j}\right\} / s_{0}^{\theta}\left(X_{j}\right)$. Under assumptions (C1)-(C5), we have almost surely

$$
\mathcal{N}_{n}^{\theta}=\mathcal{E}_{n}^{\theta}+\frac{\tilde{c}_{1, n}}{n h}+\tilde{c}_{2, n} h^{4}+\mathcal{R}_{n}^{\theta}+\mathcal{B}_{n}^{\theta}\left(\theta-\theta_{0}\right)+\mathcal{O}\left\{n^{2 \varsigma}\left(\gamma_{n}^{3}+\delta_{\theta}^{3}\right)\right\}
$$

where $\mathcal{R}_{n}^{\theta}=\mathcal{O}\left\{n^{-1}(\log n / h)^{1 / 2}+(\log n / n)^{-1 / 2} h^{2}\right\}, \theta^{\top} \mathcal{R}_{n}^{\theta}=\mathcal{O}\left\{h n^{-1}(\log n / h)^{1 / 2}+(\log n / n)^{-1 / 2} h^{3}\right\}$ and $E\left\{\mathcal{R}_{n}^{\theta} \mathcal{E}_{0}^{\theta}\right\}=\mathcal{O}\left\{(n h)^{-2}+h^{8}\right\}, \mathcal{B}_{n}^{\theta}=W_{n}^{\theta}+\mathcal{O}(1)$ with $W_{n}^{\theta}$ defined in Lemma [5.3, $\tilde{c}_{1, n}$ and $\mathcal{E}_{0}^{\theta}$ are defined in Lemma 5.2 and

$$
\tilde{c}_{2, n}=\frac{1}{4}\left(\mu_{4}-1\right) \sum_{j=1}^{n} \rho_{n}\left\{f_{\theta}\left(X_{j}\right)\right\} g^{\prime}\left(\theta_{0}^{\top} X_{j}\right) g^{\prime \prime}\left(\theta_{0}^{\top} X_{j}\right) \nu_{\theta}^{\prime \prime}\left(X_{j}\right) \text {. }
$$

Proof of Theorem 3.1 By assumption (C2), we have

$$
\sum_{n=1}^{\infty} P\left(\bigcup_{i=1}^{n}\left\{X_{i} \notin \Lambda_{n}\right\}\right) \leq \sum_{n=1}^{\infty} n P\left(X_{i} \notin \Lambda_{n}\right) \leq \sum_{n=1}^{\infty} n P\left(\left|X_{i}\right|>n^{c}\right)<\sum_{n=1}^{\infty} n n^{-6 c} E|X|^{6}<\infty
$$

for any $c>1 / 3$. It follows from the Borel-Cantelli lemma that

$$
P\left(\bigcap_{n=1}^{\infty} \bigcup_{i=1}^{n}\left\{X_{i} \notin \Lambda_{n}\right\}\right)=0
$$

Let $\tilde{\Lambda}_{n}=\left\{x: f_{\theta}\left(\theta^{\top} x\right)>2 n^{-\epsilon}\right\}$. Similarly, we have

$$
P\left(\bigcap_{n=1}^{\infty} \bigcup_{i=1}^{n}\left\{X_{i} \notin \tilde{\Lambda}_{n}\right\}\right)=0
$$

Thus, we can exchange summations over $\left\{X_{j}: j=1, \cdots, n\right\},\left\{X_{j}: X_{j} \in \Lambda_{n}, j=1, \cdots, n\right\}$ and $\left\{X_{j}: X_{j} \in\right.$ $\left.\tilde{\Lambda}_{n}, j=1, \cdots, n\right\}$ in the sense of almost surely consistency. On the other hand, we have by (C2)

$$
n^{-1} \sum_{\left|X_{j}\right|<n^{c}}\left(1+\left|X_{j}\right|^{6}\right)=O(1)
$$


By the notation in Lemmas 5.3 and [5.4, after one iteration of Steps 1-3, the new $\theta$ is

$$
\tilde{\theta}=\theta_{0}+\left(\mathcal{D}_{n}^{\theta}\right)^{-1} \mathcal{N}_{n}^{\theta}
$$

Note that $\theta^{\top} \mathcal{E}_{n}^{\theta}=0, \theta^{\top} c_{1, n}^{\theta}=0, \theta^{\top} c_{2, n}^{\theta}=0, \theta^{\top} W_{n}^{\theta}=0, W_{n}^{\theta}\left(W_{n}^{\theta}\right)^{+}=I-\theta \theta^{\top}$ and $\delta_{\theta} / h^{2} \rightarrow 0$. We have

$$
\begin{aligned}
\tilde{\theta}= & \theta_{0}+\theta\left[\theta^{\top} d_{11}^{\theta} h^{-2}\left\{\mathcal{R}_{n}^{\theta}+\mathcal{B}_{n}^{\theta}\left(\theta-\theta_{0}\right)+\mathcal{O}\left\{n^{2 \varsigma}\left(\gamma_{n}^{3}+\delta_{\theta}^{3}\right)\right\}\right\}-d_{12}^{\theta} B^{\top} h^{-1} \mathcal{N}_{n}^{\theta}\right] \\
& -B\left(d_{12}^{\theta}\right)^{\top} \theta^{\top} h^{-1}\left[\mathcal{R}_{n}^{\theta}+\mathcal{B}_{n}^{\theta}\left(\theta-\theta_{0}\right)+\mathcal{O}\left\{n^{2 \varsigma}\left(\gamma_{n}^{3}+\delta_{\theta}^{3}\right)\right\}\right]+B d_{22}^{\theta} B^{\top} \mathcal{N}_{n}^{\theta} \\
= & \left(1+a_{n}\right) \theta_{0}+\left\{\frac{1}{2}\left(I-\theta_{0} \theta_{0}^{\top}\right)+b_{n}\right\}\left(\theta-\theta_{0}\right)+\frac{1}{2}\left\{W_{n}^{\theta}\right\}^{+} \mathcal{E}_{n}^{\theta}+\mathcal{O}\left(h^{4}\right),
\end{aligned}
$$

where $a_{n}=\mathcal{O}(1)$ and $b_{n}=\mathcal{O}(1)$.

By (25) below, we have $s_{0}^{\theta}(x)=f_{\theta}\left(\theta^{\top} x\right)+\mathcal{O}\left(\gamma_{n}\right)$. Thus by the smoothness of $\rho_{n}($.$) and (10), we have$

$$
\rho_{n}\left(s_{0}^{\theta}(x)\right)=\rho_{n}\left(f_{\theta}\left(\theta^{\top} x\right)\right)+\mathcal{O}\left(n^{\varsigma} \gamma_{n}\right)=1+\mathcal{O}\left(n^{\varsigma} \gamma_{n}\right)
$$

Since $\rho_{n}($.$) is bounded, we have E\left\{\rho_{n}\left(\hat{f}_{\theta}\left(\theta^{\top} x\right)\right)-1\right\}^{2}=\mathcal{O}(1)$. By (C3) and Lemma 6.1, we have

$$
\mathcal{E}_{n}^{\theta}=n^{-1} \sum_{i=1}^{n} g^{\prime}\left(\theta_{0}^{\top} X_{i}\right) \nu_{\theta_{0}}\left(X_{j}\right) \varepsilon_{i}+\mathcal{O}\left(n^{-1 / 2}\right) .
$$

Note that $W_{n}=W_{0}+\mathcal{O}\left(\delta_{\theta}\right)$. It is easy to check that $|\tilde{\theta}|=1+a_{n}+b_{n}+\mathcal{O}\left(h^{4}\right)=1+\mathcal{O}(1)$. Thus

$$
\tilde{\theta} /|\tilde{\theta}|=\theta_{0}+\left\{\frac{1}{2}\left(I-\theta_{0} \theta_{0}^{\top}\right)+\mathcal{O}(1)\right\}\left(\theta-\theta_{0}\right)+\frac{1}{2} n^{-1} W_{0}^{+} \sum_{i=1}^{n} g^{\prime}\left(\theta_{0}^{\top} X_{i}\right) \nu_{\theta_{0}}\left(X_{i}\right) \varepsilon_{i}+\mathcal{O}\left(h^{3}+n^{-1 / 2}\right) .
$$

Let $\theta^{(k)}$ be the value of $\theta$ after $k$ iteration. Because $h_{k+1}=\max \left\{h_{k} / c_{h}, h\right\}$. Therefore,

$$
\left|\theta_{k+1}-\theta_{0}\right| / h_{k+1}^{2} \rightarrow 0
$$

for all $k>1$. We have

$$
\theta^{(k+1)}=\theta_{0}+\left\{\frac{1}{2}\left(I-\theta_{0} \theta_{0}^{\top}\right)+\mathcal{O}(1)\right\}\left(\theta^{(k)}-\theta_{0}\right)+\frac{1}{2} n^{-1} W_{0}^{+} \sum_{i=1}^{n} g^{\prime}\left(\theta_{0}^{\top} X_{i}\right) \nu_{\theta_{0}}\left(X_{i}\right) \varepsilon_{i}+\mathcal{O}\left(h_{k}^{3}+n^{-1 / 2}\right) .
$$

Recursing the above equation, we have

$$
\begin{aligned}
\theta^{(k+1)}=\theta_{0}+\left\{\frac{1}{2^{k}}(\right. & \left.\left.I-\theta_{0} \theta_{0}^{\top}\right)+\mathcal{O}(1) \sum_{\iota=1}^{k} \frac{1}{2^{\iota}}\right\}\left(\theta^{(1)}-\theta_{0}\right)+\left\{\sum_{\iota=1}^{k} \frac{1}{2^{\iota}}\right\} n^{-1} W_{0}^{+} \sum_{i=1}^{n} g^{\prime}\left(\theta_{0}^{\top} X_{i}\right) \nu_{\theta_{0}}\left(X_{i}\right) \varepsilon_{i} \\
& +\mathcal{O}\left(\sum_{\iota=1}^{k} \frac{1}{2^{\iota}} h_{k-\iota}^{3}+n^{-1 / 2}\right) .
\end{aligned}
$$


Thus as the number of iterations $k \rightarrow \infty$, Theorem 3.1 follows immediately from the above equation and the central limit theorem.

Proof of Theorem 3.3 Based on Theorem 3.2, we can assume $\delta_{\theta}=(\log n / n)^{1 / 2}$. Note that $\theta^{\top}\left\{\mathcal{E}_{n}^{\theta}+\right.$ $\left.c_{1, n}(n h)^{-1}+c_{2, n} h^{4}\right\}=0$. We consider the product of each term in $\left(\mathcal{D}_{n}^{\theta}\right)^{-1}$ with $\mathcal{N}_{n}^{\theta}$. We have

$$
\begin{gathered}
\theta \theta^{\top} d_{11}^{\theta} h^{-2} \mathcal{N}_{n}^{\theta}=\theta \theta^{\top} d_{11}^{\theta} h^{-2}\left[\mathcal{R}_{n}^{\theta}+B_{n}^{\theta}\left(\theta-\theta_{0}\right)+\mathcal{O}\left\{n^{2 \varsigma}\left(\gamma_{n}^{3}+\delta_{\theta}^{3}\right)\right\}\right]=a_{n}^{\theta} \theta_{0}+a_{n}^{\theta}\left(\theta-\theta_{0}\right) \\
\theta d_{12}^{\theta} B^{\top} h^{-1} \mathcal{N}_{n}^{\theta}=b_{n}^{\theta} \theta_{0}+b_{n}^{\theta}\left(\theta-\theta_{0}\right), \quad B\left(d_{12}^{\theta}\right)^{\top} \theta^{\top} h^{-1} \\
\left(\mathcal{D}_{n}^{\theta}\right)^{-1} \mathcal{N}_{n}=\theta\left\{S_{n}^{\top}\left(\theta-\theta_{0}\right)+H_{n} \mathcal{E}_{n}^{\theta}+\mathcal{O}\left(n^{2 \varsigma} \gamma_{n}^{4}\right)\right\} \\
=\theta_{0}\left\{S_{n}^{\top}\left(\theta-\theta_{0}\right)+H_{n} \mathcal{E}_{0}^{\theta}+\mathcal{O}\left(n^{2 \varsigma} \gamma_{n}^{4}\right)\right\}+c_{n}\left(\theta-\theta_{0}\right)
\end{gathered}
$$

where $S_{n}=\mathcal{O}(1)$ and $c_{n}=\mathcal{O}\left(\gamma_{n} / h\right)$. It is easy to see that $c_{n}=\mathcal{O}(1)$ providing that $\left|\theta-\theta_{0}\right| / h^{2} \rightarrow 0$. By Lemma 5.3 and 5.4, we have

$$
\begin{aligned}
\tilde{\theta}= & \theta_{0}\left\{1+S_{n}^{\top}\left(\theta-\theta_{0}\right)+H_{n} \mathcal{E}_{0}^{\theta}+\mathcal{O}\left(n^{2 \varsigma} \gamma_{n}^{4}\right)\right\}+\frac{1}{2} W_{n}^{\theta}\left\{\mathcal{E}_{0}^{\theta}+\frac{c_{1, n}^{\prime}}{n h}+c_{2, n}^{\prime} h^{4}+\mathcal{R}_{n}^{\theta}+\mathcal{Q}_{n}^{\theta}\right\} \\
& +\left\{\frac{1}{2}\left(I-\theta \theta^{\top}\right)+c_{n}\right\}\left(\theta-\theta_{0}\right)+\mathcal{O}\left\{n^{2 \varsigma}\left(\gamma_{n}^{3}+h \log n / n\right)\right\} .
\end{aligned}
$$

It is easy to see that $|\tilde{\theta}|=1+S_{n}^{\top}\left(\theta-\theta_{0}\right)+H_{n} \mathcal{E}_{0}^{\theta}+\mathcal{O}\left(n^{2 \varsigma} \gamma_{n}^{4}\right)$. Thus

$\tilde{\theta} /|\tilde{\theta}|=\theta_{0}+\frac{1}{2} W_{n}^{\theta}\left\{\mathcal{E}_{0}^{\theta}+\frac{c_{1, n}^{\prime}}{n h}+c_{2, n}^{\prime} h^{4}+\mathcal{R}_{n}^{\theta}+\mathcal{E}_{0}^{\theta} H_{n}^{\top} \mathcal{E}_{0}^{\theta}\right\}+\left\{\frac{1}{2}\left(I-\theta \theta^{\top}\right)+c_{n}^{\prime}\right\}\left(\theta-\theta_{0}\right)+\mathcal{O}\left\{n^{2 \varsigma}\left(\gamma_{n}^{3}+h \log n / n\right)\right\}$,

where $c_{n}^{\prime}=\mathcal{O}(1)$. Similar to the proof of Theorem 3.1, we complete the proof with $c_{1, n}=W_{n}^{-1} c_{1, n}^{\prime}$ and $c_{2, n}=W_{n}^{-1} c_{2, n}^{\prime}$

\section{Proofs of the Lemmas}

In this section, we first give some results about the uniform consistency. Based on these results, the Lemmas are proved.

Lemma 6.1 Suppose $G_{n, i}(\chi)$ is a martingale with respect to $\mathcal{F}_{i}=\sigma\left\{G_{n, \ell}(\chi), \ell \leq i\right\}$ with $\chi \in \mathcal{X}$ and $\mathcal{X}$ is a compact region in a multidimensional space such that $(I)\left|G_{n, i}(\chi)\right|<\xi_{i}$, where $\xi_{i}$ are IID and $\sup E \xi_{1}^{2 r}<\infty$ for some $r>2$; (II) $E G_{n, k}^{2}(\chi)<a_{n} s(\chi)$ with $\inf s(\chi)$ positive, and (III) $\left|G_{n, i}(\chi)-G_{n, i}(\tilde{\chi})\right|<n^{\alpha_{1}}|\chi-\tilde{\chi}| M_{i}$, where $M_{i}, i=1,2, \ldots$ are IID with $E M_{1}^{2}<\infty$. If and $a_{n}=c n^{-\delta}$ with $0 \leq \delta<1-2 / r$, then for any $\alpha_{1}^{\prime}>0$ we have

$$
\sup _{|\chi| \leq n^{\alpha_{1}^{\prime}}}\left|n^{-1} s^{-1 / 2}(\chi) \sum_{i=1}^{n} G_{n, i}(\chi)\right|=O\left\{\left(n^{-1} a_{n} \log n\right)^{1 / 2}\right\}
$$


almost surely. Suppose for any fixed $n$ and $k, G_{n, i, k}(\theta)$ is a martingale with respect to $\mathcal{F}_{i, k}=\sigma\left\{G_{n, \ell, k}(\theta), \ell \leq\right.$ $i\}$ such that (I) $\left|G_{n, i, k}(\theta)\right| \leq \xi_{i}$, (II) $E G_{n, i, k}^{2}(\theta)<a_{n}$ and (III) $\left|G_{n, i, k}(\theta)-G_{n, i, k}(\tilde{\theta})\right|<n^{\alpha_{2}}|\theta-\tilde{\theta}| M_{i}$, where $\xi_{i}, a_{n}$ and $M_{i}$ are defined above. If $E\left|\varepsilon_{k}\right|^{2 r}<\infty$ and $E\left\{\varepsilon_{k} \mid G_{n, i, j}(\theta), i<j, j=1, \ldots, k-1\right\}=0$, then

$$
\sup _{\theta \in \Theta}\left|n^{-2} \sum_{k=2}^{n}\left\{\sum_{i=1}^{k-1} G_{n, i, k}(\theta)\right\} \varepsilon_{k}\right|=O\left\{\left(a_{n} \log n\right)^{1 / 2} / n\right\}
$$

almost surely.

Proof of Lemma 6.1 We give the details for the second part of the Lemma. The first part is easier and can be proved similarly. Let $\Delta_{n}(\theta)$ be the expression between the absolute symbols in the equation. By (III) and the strong low of large numbers, it is easy to see that there are $n_{1}=n^{\alpha_{3}}$ balls centered at $\theta_{\iota}: B \iota=\left\{\theta:\left|\theta-\theta_{\iota}\right|<n^{-\alpha_{4}}\right\}$ with $\alpha_{4}>\alpha_{2}+2$, such that $\bigcup_{\iota=1}^{n_{1}} B \iota \supset \Theta$. By the strong law of large numbers, we have

$$
\max _{1 \leq \iota \leq n_{1}} \sup _{\theta \in B \iota}\left|\Delta_{n}(\theta)-\Delta_{n}(\theta \iota)\right| \leq n^{\alpha_{2}} \max _{1 \leq \iota \leq n_{1}} \sup _{\theta \in B \iota}\left|\theta-\theta_{\iota}\right| n^{-2} \sum_{k=1}^{n}\left|\varepsilon_{k}\right| \sum_{i=1}^{n} M_{i}=O\left\{\left(a_{n} \log n\right)^{1 / 2} / n\right\}
$$

almost surely. Let $\Delta_{n, k}(\theta \iota)=\sum_{i=1}^{k-1} G_{n, i, k}(\theta \iota)$. Next, we show that there is a constant $c_{1}$ such that

$$
p_{n} \stackrel{\text { def }}{=} P\left(\bigcap_{\ell=1}^{\infty} \bigcup_{n=\ell}^{\infty}\left\{\max _{1<k \leq n} \max _{1<\iota \leq n_{1}}\left|\Delta_{n, k}(\theta \iota)\right|>c_{1}\left(n a_{n} \log n\right)^{1 / 2}\right\}\right)=0 .
$$

Let $T_{n}=\left\{n a_{n} \log (n)\right\}^{1 / 2}, G_{n, i, k}^{I}(\theta \iota)=G_{n, i, k}(\theta \iota) I\left(\left|G_{n, i, k}(\theta \iota)\right| \leq T_{n}\right)$ and $G_{n, i, k}^{O}(\theta \iota)=G_{n, i, k}(\theta \iota)-G_{n, i, k}^{I}(\theta \iota)$. Write

$$
\Delta_{n, k}(\theta \iota)=\sum_{i=1}^{k-1}\left\{G_{n, i, k}^{I}(\theta \iota)-E G_{n, i, k}^{I}(\theta \iota)\right\}+\sum_{i=1}^{k-1}\left\{G_{n, i, k}^{O}(\theta \iota)-E G_{n, i, k}^{O}(\theta \iota)\right\} .
$$

Note that $E\left|G_{n, i, k}^{O}(\theta \iota)\right| \leq T_{n}^{-r+1} E\left|\xi_{1}\right|^{r}=E\left|\xi_{1}\right|^{r}\left\{n a_{n} \log (n)\right\}^{-(r-1) / 2}$. If $a_{n}=c n^{-\delta}$ with $0 \leq \delta<1-2 / r$ and $k \leq n$, we have

$$
\left|\sum_{i=1}^{k-1} E G_{n, i, k}^{O}(\theta \iota)\right| \leq E\left|\xi_{1}\right|^{r}(k-1)\left\{n a_{n} \log (n)\right\}^{-(r-1) / 2} \leq C E\left|\xi_{1}\right|^{r}\left\{n a_{n} \log (n)\right\}^{1 / 2} .
$$

Note that

$$
\sum_{i=1}^{n}\left|G_{n, i, k}^{O}(\theta \iota)\right| \leq \sum_{i=1}^{n}\left|\xi_{i}\right| I\left(\left|\xi_{i}\right|>T_{n}\right) \leq T_{n}^{-r+1} \sum_{i=1}^{n}\left|\xi_{i}\right|^{r} I\left(\left|\xi_{i}\right|>T_{n}\right)
$$

For fixed $T$, by the strong law of large numbers, we have

$$
n^{-1} \sum_{i=1}^{n}\left|\xi_{i}\right|^{r} I\left(\left|\xi_{i}\right|>T\right) \rightarrow E\left\{\left|\xi_{1}\right|^{r} I\left(\left|\xi_{1}\right|>T\right)\right\}
$$


almost surely. The right hand side above is dominated by $E\left\{\left|\xi_{1}\right|^{r}\right\}$ and $\rightarrow 0$ as $T \rightarrow \infty$. Note that $T_{n}$ increase to $\infty$ with $n$. For large $n$ such that $T_{n}>T$, we have

$$
n^{-1} \sum_{i=1}^{n}\left|\xi_{i}\right|^{r} I\left(\left|\xi_{i}\right|>T_{n}\right) \leq n^{-1} \sum_{i=1}^{n}\left|\xi_{i}\right|^{r} I\left(\left|\xi_{i}\right|>T\right) \rightarrow 0
$$

almost surely as $T \rightarrow \infty$. It follows

$$
\sum_{i=1}^{n}\left|G_{n, i, k}^{O}(\theta \iota)\right|=o\left(n T_{n}^{-r+1}\right)=o\left\{\left(n a_{n} \log n\right)^{1 / 2}\right\}
$$

almost surely. Thus by (15) and (16), if $c_{1}^{\prime}>C E\left|\xi_{1}\right|^{r}$ we have

$$
\begin{aligned}
& p_{n}^{\prime} \stackrel{\text { def }}{=} P\left(\bigcap_{\ell=1}^{\infty} \bigcup_{n=\ell}^{\infty}\left\{\max _{1<k \leq n} \max _{1<\iota \leq n_{1}}\left|\sum_{i=1}^{k-1}\left\{G_{n, i, k}^{O}(\theta \iota)-E G_{n, i, k}^{O}(\theta \iota)\right\}\right|>c_{1}^{\prime}\left(n a_{n} \log n\right)^{1 / 2}\right\}\right) \\
& \leq P\left(\bigcap_{\ell=1}^{\infty} \bigcup_{n=\ell}^{\infty}\left\{\sum_{i=1}^{n}\left|\xi_{i}\right|\left(\left|\xi_{i}\right| \geq T_{n}\right)>c_{1}^{\prime}\left(n a_{n} \log n\right)^{1 / 2}\right\}\right) \\
& \quad+P\left(\bigcap_{\ell=1}^{\infty} \bigcup_{n=\ell}^{\infty}\left\{\max _{1<k \leq n} \max _{1<\iota \leq n_{1}}\left|\sum_{i=1}^{k-1} E G_{n, i, k}^{O}(\theta \iota)\right|>c_{1}^{\prime}\left(n a_{n} \log n\right)^{1 / 2}\right\}\right) \\
& =0 .
\end{aligned}
$$

By condition (II), if $k \leq n$ we have

$$
\max _{1 \leq \iota \leq n_{1}} \operatorname{Var} \sum_{i=1}^{k-1}\left\{G_{n, i, k}^{I}(\theta \iota)-E G_{n, i, k}^{I}(\theta \iota)\right\} \leq c_{2} n a_{n} \stackrel{\text { def }}{=} N_{1},
$$

where $c_{2}$ is a constant. By the condition on $a_{n}$ and the definition of $G_{n, i, k}^{I}(\theta \iota)$, we have constants $c_{3}$ and $c_{4}$ such that

$$
\begin{aligned}
\max _{1 \leq \iota \leq n^{\alpha}}\left|\left\{G_{n, i, k}^{I}(\theta \iota)-E G_{n, i, k}^{I}(\theta \iota)\right\}\right| & \leq c_{3} T_{n} \\
& =c_{3}\left\{n a_{n} / \log n\right\}^{1 / 2}\left\{a_{n}^{-r} \log ^{r+1} n / n^{r-2}\right\}^{1 /(2(r-1))} \\
& \leq c_{4}\left\{n a_{n} / \log n\right\}^{1 / 2} \stackrel{\text { def }}{=} N_{2} .
\end{aligned}
$$

Let $N_{3}=c_{5}\left\{n a_{n} \log n\right\}^{1 / 2}$ with $c_{5}^{2}>2\left(\alpha_{3}+3\right)\left(c_{2}+c_{4} c_{5}\right)$. By the Bernstein's inequality (cf. DE LA Peña, 1999), we have from (18) and (19) that for any $k \leq n$,

$$
\begin{aligned}
P\left(\left|\sum_{i=1}^{k-1}\left\{G_{n, i, k}^{I}(\theta \iota)-E G_{n, i, k}^{I}(\theta \iota)\right\}\right|>N_{3}\right) & \leq 2 \exp \left(\frac{-N_{3}^{2}}{2\left(N_{1}+N_{2} N_{3}\right)}\right) \\
& \leq 2 \exp \left\{-c_{5}^{2} \log n /\left(2 c_{2}+2 c_{4} c_{5}\right)\right\} \\
& \leq c_{6} n^{-\alpha_{3}-3} .
\end{aligned}
$$


Let $c_{1}>\max \left\{c_{5}, c_{1}^{\prime}\right\}$. We have

$$
\begin{aligned}
& \sum_{n=1}^{\infty} P\left\{\max _{1<k \leq n} \max _{1<\iota \leq n_{1}}\left|\sum_{i=1}^{k-1}\left[G_{n, i, k}^{I}(\theta \iota)-E G_{n, i, k}^{I}(\theta \iota)\right]\right|>c_{1}\left(n a_{n} \log n\right)^{1 / 2}\right\} \\
& \leq \sum_{n=1}^{\infty} \sum_{k=2}^{n} \sum_{\iota=1}^{n_{1}} P\left\{\left|\sum_{i=1}^{k-1}\left[G_{n, i, k}^{I}(\theta \iota)-E G_{n, i, k}^{I}(\theta \iota)\right]\right|>c_{1}\left(n a_{n} \log n\right)^{1 / 2}\right\} \\
& \leq \sum_{n=1}^{\infty} c_{6} n^{-\alpha_{3}-3} n^{1+\alpha_{3}}<\infty .
\end{aligned}
$$

By (14), (17) and (20) and the Borel-Cantelli lemma, we have

$$
p_{n} \leq P\left\{\bigcap_{\ell=1}^{\infty} \bigcup_{n=\ell}^{\infty} \max _{1<k \leq n} \max _{1<\iota \leq n_{1}}\left|\sum_{i=1}^{k-1}\left[G_{n, i, k}^{I}(\theta \iota)-E G_{n, i, k}^{I}(\theta \iota)\right]\right|>c_{1}\left(n a_{n} \log n\right)^{1 / 2}\right\}+p_{n}^{\prime}=0 .
$$

Therefore (13) follows.

Let $\Delta_{n, k}^{I}\left(\theta_{\iota}\right)=\Delta_{n, k}\left(\theta_{\iota}\right) I\left\{\left|\Delta_{n, k}\left(\theta_{\iota}\right)\right| \leq c_{1}\left(n a_{n} \log n\right)^{1 / 2}\right\}$ and $U_{\ell}\left(\theta_{\iota}\right)=\sum_{k=2}^{\ell} \Delta_{n, k}^{I}\left(\theta_{\iota}\right) \varepsilon_{k}$. Write

$$
\Delta_{n}\left(\theta_{\iota}\right)=U_{n}\left(\theta_{\iota}\right)+\sum_{k=2}^{n} \Delta_{n, k}^{O}\left(\theta_{\iota}\right) \varepsilon_{k}
$$

where $\Delta_{n, k}^{O}\left(\theta_{\iota}\right)=\Delta_{n, k}\left(\theta_{\iota}\right)-\Delta_{n, k}^{I}\left(\theta_{\iota}\right)$. It is easy to see from (13) that for the second part on the right hand side above,

$$
\max _{1<\iota \leq n_{1}}\left|\sum_{k=2}^{n} \Delta_{n, k}^{O}\left(\theta_{\iota}\right) \varepsilon_{k}\right|=O\left\{n\left(a_{n} \log n\right)^{1 / 2}\right\}
$$

almost surely, since for any constant $c>0$,

$$
\begin{aligned}
\sum_{n=1}^{\infty} P\left\{\max _{1<\iota \leq n_{1}}\left|\sum_{k=2}^{n} \Delta_{n, k}^{O}\left(\theta_{\iota}\right) \varepsilon_{k}\right|>c n\left(a_{n} \log n\right)^{1 / 2}\right\} & \leq \sum_{n=1}^{\infty} P\left(\max _{1<\iota \leq n_{1}} \max _{1<k \leq n}\left|\Delta_{n, k}^{O}\left(\theta_{\iota}\right)\right|>0\right) \\
& \leq \sum_{n=1}^{\infty} P\left\{\max _{1<\iota \leq n} \max _{1<k \leq n}\left|\Delta_{n, k}\left(\theta_{\iota}\right)\right|>c_{1}\left(n a_{n} \log n\right)^{1 / 2}\right\} \\
& <\infty .
\end{aligned}
$$

Now consider the first term. Let $T_{n}^{1 / 2} / \log n$,

$$
U_{\ell}^{I}\left(\theta_{\iota}\right)=\sum_{k=2}^{\ell} \Delta_{n, k}^{I}\left(\theta_{\iota}\right)\left\{\varepsilon_{k}\left(\left|\varepsilon_{k}\right| \leq T_{n}^{\prime}\right)-E\left[\varepsilon_{k}\left(\left|\varepsilon_{k}\right| \leq T_{n}^{\prime}\right)\right]\right\}
$$

and $U_{\ell}^{O}\left(\theta_{\iota}\right)=U_{\ell}\left(\theta_{\iota}\right)-U_{\ell}^{I}\left(\theta_{\iota}\right)$. Similar to the proof of (15) and (16), we have almost surely

$$
\begin{gathered}
\left|\sum_{k=2}^{\ell} \Delta_{n, k}^{O}\left(\theta_{\iota}\right) E\left\{\varepsilon_{k}\left(\left|\varepsilon_{k}\right|>T_{n}^{\prime}\right)\right\}\right|=O\left\{n\left(a_{n} \log n\right)^{1 / 2}\right\}, \\
\left|\sum_{k=2}^{\ell} \Delta_{n, k}^{O}\left(\theta_{\iota}\right) \varepsilon_{k}\left(\left|\varepsilon_{k}\right|>T_{n}^{\prime}\right)\right|=O\left\{n\left(a_{n} \log n\right)^{1 / 2}\right\} .
\end{gathered}
$$


Note that

$$
\left|\Delta_{n, k}^{I}\left(\theta_{\iota}\right)\left\{\varepsilon_{k}\left(\left|\varepsilon_{k}\right| \leq T_{n}^{\prime}\right)-E\left[\varepsilon_{k}\left(\left|\varepsilon_{k}\right| \leq T_{n}^{\prime}\right)\right]\right\}\right|<2 c_{1}\left(n a_{n} \log n\right)^{1 / 2} T_{n}^{\prime}=2 c_{1} n\left(a_{n} / \log n\right)^{1 / 2} \stackrel{\text { def }}{=} N_{4}
$$

and by (II), $\operatorname{Var}\left\{U_{\ell}^{I}(\theta \iota)\right\}=c_{2}^{\prime 2} a_{n} \stackrel{\text { def }}{=} N_{5}$, where $c_{2}^{\prime}$ is a constant. Let $N_{6}=c_{3}^{\prime} n\left(a_{n} \log n\right)^{1 / 2}$ with $c_{3}^{\prime 2}>$ $2\left(\alpha_{3}+3\right)\left(2 c_{1} c_{3}^{\prime}+c_{2}^{\prime}\right)$. By the Berenstein's inequality, we have

$$
P\left(\left|U_{n}^{I}\left(\theta_{\iota}\right)\right| \geq N_{6}\right) \leq 2 \exp \left\{-\frac{N_{6}^{2}}{2\left(N_{6} N_{4}+N_{5}\right)}\right\} \leq 2 n^{-\alpha_{3}-3} .
$$

Therefore

$$
\sum_{n=1}^{n} P\left\{\max _{1 \leq \iota \leq n_{1}}\left|U_{n}^{I}\left(\theta_{\iota}\right)\right| \geq N_{6}\right\}<\sum_{n=1}^{n} n_{1} P\left\{\left|U_{n}^{I}\left(\theta_{\iota}\right)\right| \geq N_{6}\right\}<\infty .
$$

By the Borel-Cantelli lemma, we have

$$
\max _{1 \leq \iota \leq n_{1}}\left|U_{n}^{I}\left(\theta_{\iota}\right)\right|=O\left(N_{6}\right)
$$

almost surely. Lemma 6.1 follows from (21), (22), (23) and (24).

Proof of Lemma 5.1 Write $s_{k}^{\theta}(x)=\epsilon_{k}^{\theta}(x)+E s_{k}^{\theta}(x)$. By Taylor expansion, we have

$$
s_{k}^{\theta}(x)=\sum_{\tau=0}^{3} \mu_{k+\tau} f_{\theta}^{(\tau)}(x) h^{\tau}+\epsilon_{k}^{\theta}(x)+\mathcal{O}\left(h^{4}\right) .
$$

Because $\operatorname{Var}\left\{\epsilon_{k}^{\theta}(x)\right\}=O\left\{(n h)^{-1}\right\}$, it follows from Lemma 6.1 that $\epsilon_{k}^{\theta}(x)=\mathcal{O}\left(\delta_{n}\right)$. It is easy to check that

$$
\begin{gathered}
D_{n, 0}^{\theta}(x)=f_{\theta}^{2}+\frac{1}{2}\left(\mu_{4}+1\right) f_{\theta} f_{\theta}^{\prime \prime} h^{2}-\left(f_{\theta}^{\prime}\right)^{2} h^{2}+f\left(\epsilon_{0}^{\theta}+\epsilon_{2}^{\theta}\right)-2 f_{\theta}^{\prime} h \epsilon_{1}^{\theta}+\mathcal{O}\left(\gamma_{n}^{2}\right) . \\
D_{n, 2}^{\theta}(x)=f_{\theta}^{2}+\mu_{4}\left(f_{\theta} f_{\theta}^{\prime \prime}-\left(f_{\theta}^{\prime}\right)^{2}\right) h^{2}+2 f_{\theta} \epsilon_{2}^{\theta}-f_{\theta}^{\prime} h \epsilon_{3}^{\theta}-\mu_{4} f_{\theta}^{\prime} h \epsilon_{1}^{\theta}+\mathcal{O}\left(\gamma_{n}^{2}\right) . \\
D_{n, 3}^{\theta}(x)=f_{\theta} \epsilon_{3}^{\theta}+\mathcal{O}\left(h \gamma_{n}\right), \quad D_{n, 4}^{\theta}(x)=\mu_{4} f_{\theta}^{2}+\mathcal{O}\left(\gamma_{n}\right), \quad D_{n, 5}^{\theta}(x)=\mathcal{O}(h) . \\
T_{n, 0}^{\theta}(X \mid x)=f_{\theta}^{2} \nu_{\theta}(x)+\mathcal{O}\left(\gamma_{n}\right), \quad S_{n, 0}^{\theta}(X \mid x)=\mathcal{O}(h), \quad T_{n, k}^{\theta}(X \mid x)=\mathcal{O}(1), \quad S_{n, k}^{\theta}(X \mid x)=\mathcal{O}(1), \text { for } k \geq 1, \\
T_{n, 0}^{\theta}\left(\left|\theta^{\top} X_{i x}\right|^{6} \mid x\right)=\mathcal{O}\left(h^{6}\right), \quad S_{n, 0}^{\theta}\left(\left|\theta^{\top} X_{i x}\right|^{6} \mid x\right)=\mathcal{O}\left(h^{6}\right), \quad T_{n, 0}^{\theta}\left(X X^{\top} \mid x\right)=\mathcal{O}(1), \quad S_{n, 0}^{\theta}\left(X X^{\top} \mid x\right)=\mathcal{O}(h), \\
E_{n, 2}(x)=\left(\mu_{4}-1\right) f_{\theta} f_{\theta}^{\prime} h+f\left(\epsilon_{3}^{\theta}-\epsilon_{1}^{\theta}\right)+\mathcal{O}\left(h \gamma_{n}\right), \quad E_{n, 3}(x)=\mu_{4} f_{\theta}^{2}+\mathcal{O}\left(\gamma_{n}\right), \quad E_{n, 4}(x)=\mathcal{O}(h) .
\end{gathered}
$$

Note that

$$
a^{\theta}(x)=T_{n, 0}^{\theta}(Y \mid x) / D_{n, 0}^{\theta}(x), \quad d^{\theta}(x) h=S_{n, 0}^{\theta}(Y \mid x) / D_{n, 0}^{\theta}(x) .
$$


and

$$
\begin{aligned}
& A_{n}^{\theta}(x)=\sum_{k=2}^{5} \frac{1}{k !} g^{(k)}\left(\theta_{0}^{\top} x\right) \frac{D_{n, k}^{\theta}(x)}{D_{n, 0}^{\theta}(x)} h^{k-2}, \quad B_{n}^{\theta}(x)=\sum_{k=0}^{4} \frac{1}{k !} g^{(k+1)}\left(\theta_{0}^{\top} x\right) \frac{T_{n, k}(X \mid x)-D_{n, k}(x) x}{D_{n, 0}^{\theta}(x)} h^{k}, \\
& C_{n}(x, \theta)=\frac{1}{2} g^{\prime \prime}\left(\theta_{0}^{\top} x\right)\left\{T_{n, 0}\left(X X^{\top} \mid x\right)-T_{n, 0}(X \mid x) x^{\top}-x T_{n, 0}\left(X^{\top} \mid x\right)+x x^{\top} D_{n, 0}^{\theta}(x)\right\}\left\{D_{n, 0}^{\theta}(x)\right\}^{-1}, \\
& \tilde{A}_{n}^{\theta}(x)=\sum_{k=2}^{4} \frac{1}{k !} g^{(k)}\left(\theta_{0}^{\top} x\right) \frac{E_{n, k}^{\theta}(x)}{D_{n, 0}^{\theta}(x)} h^{k-2}, \quad \tilde{B}_{n}^{\theta}(x)=\sum_{k=1}^{4} \frac{k}{k !} g^{(k)}\left(\theta_{0}^{\top} x\right) \frac{S_{n, k}(X \mid x)-E_{n, k}(x) x}{D_{n, 0}^{\theta}(x)} h^{k}, \\
& \tilde{C}_{n}(x, \theta)=\frac{1}{2} g^{\prime \prime}\left(\theta_{0}^{\top} x\right)\left\{S_{n, 0}\left(X X^{\top} \mid x\right)-S_{n, 0}(X \mid x) x^{\top}-x S_{n, 0}\left(X^{\top} \mid x\right)+x x^{\top} E_{n, 0}^{\theta}(x)\right\}\left\{D_{n, 0}^{\theta}(x)\right\}^{-1} .
\end{aligned}
$$

Lemma 5.1 follows from simple calculations based on the above equations.

Proof of Lemma 5.2 It follows from Lemma 6.1 that $\eta_{n}^{\theta}(x)=\mathcal{O}\left(\delta_{n}\right)(1+|x|)$ and $s_{0}^{\theta}=f_{\theta}+\tilde{\epsilon}_{0}^{\theta}$ where $\tilde{\epsilon}_{0}^{\theta}=\epsilon_{k}^{\theta}+\left(E s_{k}^{\theta}-f_{\theta}\right)=\mathcal{O}\left(\gamma_{n}\right)$. Because $\left|\rho_{n}^{\prime \prime}().\right|<n^{2 \varsigma}$, we have

$$
\rho_{n}\left(s_{0}^{\theta}\left(X_{j}\right)\right)=\rho_{n}\left(f_{\theta}\left(X_{j}\right)\right)+\rho_{n}^{\prime}\left(f_{\theta}\left(X_{j}\right)\right) \tilde{\epsilon}_{0}^{\theta}\left(X_{j}\right)+\mathcal{O}\left(n^{2 \varsigma} \gamma_{n}^{2}\right) .
$$

Thus

$$
\mathcal{A}_{n}^{\theta}=\tilde{\mathcal{E}}_{n}^{\theta}+Q_{n, 1}^{\theta}+\mathcal{O}\left(n^{2 \varsigma} \gamma_{n}^{3}\right)
$$

where $\tilde{\mathcal{E}}_{n}^{\theta}=n^{-2} \sum_{i=1}^{n} \sum_{j=1}^{n} \rho_{n}\left(f_{\theta}\left(X_{j}\right)\right) f_{\theta}^{-1}\left(X_{j}\right) g^{\prime}\left(\theta^{\top} X_{j}\right) K_{h}^{\theta}\left(X_{i j}\right) X_{i j} \varepsilon_{i}$, and $Q_{n, 1}^{\theta}=n^{-1} \sum_{i=1}^{n} G_{n, i}^{\theta}$ with

$$
\begin{aligned}
G_{n, i}^{\theta}=n^{-1} \sum_{j=1}^{n}\left[\frac{1}{2} f_{\theta}^{\prime \prime}\left(X_{j}\right)\left\{\rho_{n}^{\prime}\left(f_{\theta}\left(X_{j}\right)\right)-\rho_{n}\left(f_{\theta}\left(X_{j}\right)\right) f_{\theta}^{-1}\left(X_{j}\right)\right\} h^{2}+\{1-\right. & \left.\rho_{n}\left(f_{\theta}\left(X_{j}\right) f_{\theta}^{-1}\left(X_{j}\right)\right\} \tilde{\epsilon}_{0}^{\theta}\left(X_{j}\right)\right] \\
& \times f_{\theta}^{-1}\left(X_{j}\right) g^{\prime}\left(\theta^{\top} X_{j}\right) K_{h}^{\theta}\left(X_{i j}\right) X_{i j} \varepsilon_{i} .
\end{aligned}
$$

Simple calculations lead to $E \tilde{\mathcal{E}}_{n}^{\theta}=0, E\left(\tilde{\mathcal{E}}_{n}^{\theta}\right)^{2}=O\left(n^{-1}\right), E\left(G_{n, i}^{\theta}\right)=0$ and $E\left(G_{n, i}^{\theta}\right)^{2}=O\left\{h^{4}+(n h)^{-1}\right\}$. By the first part of Lemma 6.1, we have

$$
\tilde{\mathcal{E}}_{n}^{\theta}=\mathcal{O}\left\{(\log n / n)^{1 / 2}\right\}, \quad Q_{n, 1}^{\theta}=\mathcal{O}\left\{h^{2}(\log n / n)^{1 / 2}+n^{-1}(\log n / h)^{1 / 2}\right\} .
$$

By Taylor expansion, $g^{\prime}\left(\theta_{0}^{\top} x\right)=g^{\prime}\left(\theta^{\top} x\right)+g^{\prime \prime}\left(v^{*}\right)\left(\theta_{0}-\theta\right)^{\top} x$, where $v^{*}$ is a value between $\theta^{\top} x$ and $\theta_{0}^{\top} x$. Write

$$
\tilde{\mathcal{E}}_{n}^{\theta}=\mathcal{E}_{n}^{\theta}+Q_{n, 2}^{\theta}+r_{n, 0}\left(\theta-\theta_{0}\right)
$$

where $Q_{n, 2}^{\theta}=n^{-2} \sum_{i=1}^{n} \sum_{j=1}^{n}\left\{\rho_{n}\left(f_{\theta}\left(X_{j}\right)\right) f_{\theta}^{-1}\left(X_{j}\right) g^{\prime}\left(\theta^{\top} X_{j}\right) K_{h}^{\theta}\left(X_{i j}\right) X_{i j}-\rho_{n}\left(f_{\theta}\left(X_{i}\right)\right) g^{\prime}\left(\theta^{\top} X_{i}\right) \nu_{\theta}\left(X_{i}\right)\right\} \varepsilon_{i}$ and $r_{n, 0}=\mathcal{O}\left(\gamma_{n} / h\right)$. By Lemma 6.1 and that $\operatorname{Var}\left(Q_{n, 2}^{\theta}\right)=O\left\{h^{4}+(n h)^{-1}\right\}$, we have

$$
Q_{n, 2}^{\theta}=\mathcal{O}\left\{(n / \log n)^{-1 / 2} \gamma_{n}\right\}
$$


Let $Q_{n}^{\theta}=Q_{n, 1}^{\theta}+Q_{n, 2}^{\theta}$. It is easy to check that $E\left\{Q_{n}^{\theta} \mathcal{E}_{n}^{\theta}\right\}=o\left(h^{8}+(n h)^{-2}\right)$. Therefore, the first part of Lemma 5.2 follows.

Similarly, we have from (26) that

$$
\mathcal{B}_{n}^{\theta}=(n h)^{-1} \sum_{j=1}^{n}\left\{\rho_{n}\left(f_{\theta}\left(X_{j}\right)\right)+\rho_{n}^{\prime}\left(f_{\theta}\left(X_{j}\right)\right) \tilde{\epsilon}_{0}^{\theta}\left(X_{j}\right)\right\} e_{k}^{\theta}\left(X_{j}\right) \eta_{n}^{\theta}\left(X_{j}\right) / f_{\theta}\left(X_{j}\right)+\mathcal{O}\left(n^{2 \varsigma} \gamma_{n}^{4} / h\right)
$$

Let $\tilde{R}_{n}^{\theta}$ be the first term on the right hand side above. Then

$$
\begin{aligned}
\tilde{R}_{n}^{\theta}= & n^{-3} \sum_{j=1}^{n}\left\{\rho_{n}\left(f_{\theta}\left(X_{j}\right)\right)+\rho_{n}^{\prime}\left(f_{\theta}\left(X_{j}\right)\right) \tilde{\epsilon}_{0}^{\theta}\left(X_{j}\right)\right\} \sum_{i=1}^{n} K_{h}^{2}\left(\theta^{\top} X_{i j}\right)\left(\theta^{\top} X_{i j} / h\right)^{k} X_{i j} \varepsilon_{i}^{2} / f_{\theta}\left(X_{j}\right) \\
& +n^{-3} \sum_{j=1}^{n}\left\{\rho_{n}\left(f_{\theta}\left(X_{j}\right)\right)+\rho_{n}^{\prime}\left(f_{\theta}\left(X_{j}\right)\right) \tilde{\epsilon}_{0}^{\theta}\left(X_{j}\right)\right\} \sum_{i \neq \ell}^{n} K_{h}\left(\theta^{\top} X_{i j}\right)\left(\theta^{\top} X_{i j} / h\right)^{k} K_{h}\left(\theta^{\top} X_{\ell j}\right) X_{\ell j} \varepsilon_{i} \varepsilon_{\ell} / f_{\theta}\left(X_{j}\right) \\
& \stackrel{\text { def }}{=} \tilde{R}_{n, 1}^{\theta}+\tilde{R}_{n, 2}^{\theta}+\tilde{R}_{n, 3}^{\theta}+\tilde{R}_{n, 4}^{\theta} .
\end{aligned}
$$

If $\varepsilon$ is independent of $X$, then

$$
E_{\theta}(x) \stackrel{\text { def }}{=} E\left\{K_{h}^{2}\left(\theta^{\top} X_{i x}\right)\left(\theta^{\top} X_{i j} / h\right)^{k} X_{i x} \varepsilon_{i}^{2}\right\}=h^{-1} \sum_{\ell=0}^{2} \frac{1}{\ell !} \tilde{\mu}_{k+\ell}\left\{f_{\theta}(x) \nu_{\theta}(x)\right\}^{(\ell)} h^{\ell} \sigma^{2}+O\left(h^{2}\right),
$$

where $\tilde{\mu}_{k}=\int K^{2}(v) v^{k} d v$. By Lemma 6.1, we have

$$
n^{-1} \sum_{i=1}^{n} K_{h}^{2}\left(\theta^{\top} X_{i x}\right)\left(\theta^{\top} X_{i x} / h\right)^{k} X_{i x} \varepsilon_{i}^{2}-E_{\theta}(x)=\mathcal{O}\left(h^{-1} \delta_{n}\right)
$$

Thus

$$
R_{n, 0}^{\theta} \stackrel{\text { def }}{=}\left(n^{2} h\right)^{-1} \sum_{j=1}^{n} \rho_{n}\left(f_{\theta}\left(X_{j}\right)\right)\left[n^{-1} \sum_{i=1}^{n} K_{h}^{2}\left(\theta^{\top} X_{i j}\right)\left(\theta^{\top} X_{i j} / h\right)^{k} X_{i j} \varepsilon_{i}^{2}-E_{\theta}\left(X_{j}\right)\right]=\mathcal{O}\left\{\left(n h^{2}\right)^{-1} \delta_{n}\right\} .
$$

It is easy to check that $E\left\{\mathcal{E}_{n}^{\theta} R_{n, 0}^{\theta}\right\}=0$. Write

$$
\left(n^{2} h\right)^{-1} \sum_{j=1}^{n} \rho_{n}\left(f_{\theta}\left(X_{j}\right)\right) E_{\theta}\left(X_{j}\right)=(n h)^{-1} E\left\{\rho_{n}\left(f_{\theta}\left(X_{j}\right)\right) E_{\theta}\left(X_{j}\right)\right\}+R_{n, 1}^{\theta},
$$

where $E\left\{R_{n, 1}^{\theta} \mathcal{E}_{n}^{\theta}\right\}=0$ and

$$
R_{n, 1}^{\theta}=\left(n^{2} h\right)^{-1} \sum_{j=1}^{n}\left[\rho_{n}\left(f_{\theta}\left(X_{j}\right)\right) E_{\theta}\left(X_{j}\right)-E\left\{\rho_{n}\left(f_{\theta}\left(X_{j}\right)\right) E_{\theta}\left(X_{j}\right)\right\}\right]=\mathcal{O}\left\{\left(n h^{2}\right)^{-1}(n / \log n)^{-1 / 2}\right\} .
$$

Note that $E\left\{\rho_{n}\left(f_{\theta}(X)\right) \nu_{\theta}(X)\right\}=0$. We have

$$
(n h)^{-1} E\left\{\rho_{n}\left(f_{\theta}\left(X_{j}\right)\right) E_{\theta}\left(X_{j}\right)\right\}=\frac{\tilde{c}_{k, n}}{n h}+R_{n, 2}^{\theta} .
$$


where $R_{n, 2}^{\theta}=O\left(n^{-1}\right)$ and $E\left\{R_{n, 2}^{\theta} \mathcal{E}_{0}^{\theta}\right\}=0$. By (27)-(29) and the fact that $(n / \log n)^{-1 / 2}=o\left(\gamma_{n}\right)$, we have

$$
\tilde{R}_{n, 1}^{\theta}=\frac{\tilde{c}_{k}}{n h}+R_{n, 1}^{\theta}+R_{n, 2}^{\theta}
$$

Similarly

$$
\tilde{R}_{n, 2}^{\theta}=\mathcal{O}\left\{(n h)^{-1} \gamma_{n}\right\}
$$

Let $G_{n, i, \ell}^{\theta}=n^{-1} \sum_{j=1}^{n} \rho_{n}\left(f_{\theta}\left(X_{j}\right)\right) K_{h}\left(\theta^{\top} X_{i j}\right)\left(\theta^{\top} X_{i j} / h\right)^{k} K_{h}\left(\theta^{\top} X_{\ell j}\right) X_{\ell j} / f_{\theta}\left(X_{j}\right)$. Write $\tilde{R}_{n, 3}^{\theta}$ as

$$
\tilde{R}_{n, 3}^{\theta}=n^{-2} \sum_{i \neq \ell}^{n} \frac{1}{2}\left(G_{n, i, \ell}^{\theta}+G_{n, \ell, i}^{\theta}\right) \varepsilon_{i} \varepsilon_{\ell}=n^{-2} \sum_{\ell=1}^{n}\left\{\sum_{i<\ell} \frac{1}{2}\left(G_{n, i, \ell}^{\theta}+G_{n, \ell, i}^{\theta}\right) \varepsilon_{i}\right\} \varepsilon_{\ell} .
$$

By the second part of Lemma 6.1, we have

$$
\tilde{R}_{n, 3}^{\theta}=\mathcal{O}\left\{n^{-1 / 2} \delta_{n}\right\}
$$

Similarly, we have

$$
\tilde{R}_{n, 4}^{\theta}=\mathcal{O}\left\{n^{-1 / 2} \delta_{n}\right\}
$$

Thus the second part of Lemma 5.2 follows from (30) and (31).

The third part of Lemma 5.2 can be proved similarly as the proof of the second part.

Proof of Lemma 5.4 By (8), Lemma 5.1 and $\theta_{0}=\theta+\left(\theta_{0}-\theta\right)$, simple calculations lead to

$$
\begin{array}{r}
Y_{i}-a_{\theta}(x)-d_{\theta}(x) \theta_{0}^{\top} X_{i x}=\varepsilon_{i}+\left\{\tilde{A}^{\theta}\left(x, X_{i}\right)-A_{n}^{\theta}(x) h^{2}\right\}+\left\{\tilde{B}^{\theta}\left(x, X_{i}\right)-B_{n}^{\theta}(x)\right\}^{\top}\left(\theta_{0}-\theta\right) \\
-V_{n}^{\theta}(x)+\mathcal{O}\left\{h^{2} \gamma_{n}^{2}+\delta_{\theta}^{3}\right\},
\end{array}
$$

where $\tilde{A}^{\theta}\left(x, X_{i}\right)=A^{\theta}\left(x, X_{i}\right)-d_{\theta}(x) \theta^{\top} X_{i x}$ and $\tilde{B}^{\theta}\left(x, X_{i}\right)=B^{\theta}\left(x, X_{i}\right)-d_{\theta}(x) X_{i x}$. It follows from the Taylor expansion that

$$
C_{n, k}^{\theta}(x) \stackrel{\text { def }}{=} n^{-1} \sum_{i=1}^{n} K_{h}^{\theta}\left(X_{i x}\right)\left(\theta^{\top} X_{i x} / h\right)^{k} X_{i x}=\sum_{\ell=0}^{5} \frac{1}{\ell !} \mu_{k+\ell}\left(f \mu_{\theta}\right)^{(\ell)} h^{\ell}+\tilde{\xi}_{k}^{\theta}+\mathcal{O}\left(h^{6}\right),
$$

where $\tilde{\xi}_{k}^{\theta}=n^{-1} \sum_{i=1}^{n}\left\{K_{h}^{\theta}\left(X_{i x}\right)\left(\theta^{\top} X_{i x} / h\right)^{k} X_{i x}-E K_{h}^{\theta}\left(X_{i x}\right)\left(\theta^{\top} X_{i x} / h\right)^{k} X_{i x}\right\}=\xi_{k}^{\theta}-x \epsilon_{k}^{\theta}$. We have

$$
\begin{aligned}
& n^{-1} \sum_{i=1}^{n} K_{h}^{\theta}\left(X_{i x}\right) X_{i x} \tilde{A}^{\theta}\left(x, X_{i}\right)=\left\{g^{\prime}\left(\theta_{0}^{\top} x\right)-d_{\theta}(x)\right\} C_{n, 1}(x) h+\sum_{k=2}^{5} \frac{1}{k !} g^{(k)}\left(\theta_{0}^{\top} x\right) C_{n, k}^{\theta}(x) h^{k} \\
& =-\frac{1}{2} g^{\prime \prime}\left(\mu_{4}-1\right) f_{\theta}^{\prime} f_{\theta}^{-1}\left(\nu_{\theta} f_{\theta}\right)^{\prime} h^{4}+\frac{1}{2} g^{\prime \prime}\left(\nu_{\theta} f_{\theta}\right)^{\prime}\left(\epsilon_{3}^{\theta}-\epsilon_{1}^{\theta}\right) h^{2}+\tilde{V}_{n}^{\theta}\left\{\left(\nu_{\theta} f_{\theta}\right)^{\prime} h+\frac{1}{6} \mu_{4}\left(\nu_{\theta} f_{\theta}\right)^{\prime \prime} h^{3}+\tilde{\xi}_{1}^{\theta}\right\} \\
& \quad+\frac{1}{2} g^{\prime \prime} h^{2}\left\{f_{\theta} \nu_{\theta}+\frac{1}{2} \mu_{4}\left(f_{\theta} \nu_{\theta}\right)^{\prime \prime} h^{2}\right\}+\frac{1}{24} g^{(4)} \mu_{4} f_{\theta} \nu_{\theta} h^{4}+\frac{1}{2} g^{\prime \prime} h^{2} \tilde{\xi}_{2}^{\theta}+\frac{1}{6} g^{\prime \prime \prime} h^{3} \tilde{\xi}_{3}^{\theta}+\mathcal{O}\left(h^{2} \gamma_{n}^{2}\right) .
\end{aligned}
$$


Thus

$$
n^{-1} \sum_{i=1}^{n} K_{h}^{\theta}\left(X_{i x}\right) X_{i x}\left\{\tilde{A}^{\theta}\left(x, X_{i}\right)-A_{n}^{\theta}(x) h^{2}\right\}=\frac{1}{4}\left(\mu_{4}-1\right) g^{\prime \prime} f_{\theta} \nu_{\theta}^{\prime \prime}+B_{n, 1}^{\theta}\left(\theta-\theta_{0}\right)+H_{2, n}^{\theta}+\mathcal{O}\left(h^{2} \gamma_{n}^{2}\right),
$$

where $B_{n, 1}^{\theta}=\left\{\left(\nu_{\theta} f_{\theta}\right)^{\prime} h+\frac{1}{6} \mu_{4}\left(\nu_{\theta} f_{\theta}\right)^{\prime \prime \prime} h^{3}+\tilde{\xi}_{1}^{\theta}\right\} B_{n}^{\theta}(x)^{\top}$ with $B_{n}^{\theta}(x)$ defined in Lemma 5.1, and

$$
\begin{aligned}
H_{2, n}^{\theta}= & \frac{1}{2} g^{\prime \prime}\left(\epsilon_{3}^{\theta}-\epsilon_{1}^{\theta}\right) h^{2}\left(\nu_{\theta} f_{\theta}\right)^{\prime}+f_{\theta}^{-1} e_{1}^{\theta}\left(f_{\theta} \nu_{\theta}\right)^{\prime} h+\frac{1}{2} f_{\theta}^{-2} f_{\theta}^{\prime \prime}\left(f \nu_{\theta}\right)^{\prime} h^{3} e_{1}^{\theta}+f_{\theta}^{-2}(f \nu)^{\prime} h\left(\epsilon_{0}^{\theta} e_{1}^{\theta}-\epsilon_{1}^{\theta} e_{0}^{\theta}\right) \\
& -f^{-2} f^{\prime}(f \nu)^{\prime} h^{2} e_{0}^{\theta}+f^{-1}(f \nu)^{\prime} h \epsilon_{0}^{\theta}\left\{-\frac{1}{2}\left(\mu_{4}+1\right) f^{-1} f_{\theta}^{\prime \prime} h^{2}-f^{-1}\left(\epsilon_{0}^{\theta}+\epsilon_{1}^{\theta}\right)+f^{-2}\left(f^{\prime}\right)^{2} h^{2}\right\} \\
& +\frac{1}{6} \mu_{4} f^{-1} e_{1}^{\theta}(f \nu)^{\prime \prime} h^{3}+f^{-1} e_{1}^{\theta} \tilde{\xi}_{1}^{\theta}-f^{-2} f^{\prime} h e_{0}^{\theta} \tilde{\xi}_{1}^{\theta}+\frac{1}{2} g^{\prime \prime} h^{2} \tilde{\xi}_{2}^{\theta}+\frac{1}{6} g^{\prime \prime \prime} h^{3} \tilde{\xi}_{3}^{\theta}-\frac{1}{2} g^{\prime \prime} h^{2} \tilde{\xi}_{0}^{\theta} \\
& -\frac{1}{2} g^{\prime \prime}\left(\theta_{0}^{\top} x\right)\left\{f_{\theta}^{-1}\left(\epsilon_{2}^{\theta}-\epsilon_{0}^{\theta}\right)+\left(2-\mu_{4}\right) f_{\theta}^{-2} f_{\theta}^{\prime} h \epsilon_{1}^{\theta}-f_{\theta}^{-2} f_{\theta}^{\prime} h \epsilon_{3}^{\theta}\right\} \nu_{\theta} f_{\theta} h^{2}-\frac{1}{6} g^{\prime \prime \prime} \epsilon_{3}^{\theta} \nu_{\theta} h^{3} .
\end{aligned}
$$

By the expansions of $d_{\theta}(x)$ in Lemma [5.1, $\rho_{n}\left(s_{0}^{\theta}(x)\right)$ in (26) , and (34), we have

$$
\begin{aligned}
n^{-2} \sum_{j=1}^{n} \rho_{n}\left(s_{0}^{\theta}\left(X_{j}\right)\right) d_{\theta}\left(X_{j}\right) \sum_{i=1}^{n} K_{h}^{\theta}\left(X_{i x}\right) X_{i j}\{ & \left.\tilde{A}^{\theta}\left(X_{j}, X_{i}\right)-A_{n}^{\theta}\left(X_{j}\right) h^{2}\right\} / s_{0}^{\theta}\left(X_{j}\right) \\
& =\tilde{c}_{2, n} h^{4}+\left(B_{n, 2}^{\theta}\right)^{\top}\left(\theta-\theta_{0}\right)+\tilde{R}_{n, 1}^{\theta}+\mathcal{O}\left\{n^{2 \varsigma}\left(h^{2} \gamma_{n}^{2}+\delta_{\theta}^{2} h+\delta_{\theta}^{3}\right)\right\}
\end{aligned}
$$

where $B_{n, 2}^{\theta}=n^{-1} \sum_{j=1}^{n} \rho_{n}\left(s_{0}^{\theta}\left(X_{j}\right)\right) d_{\theta}\left(X_{j}\right) B_{1, n}^{\theta}\left(X_{j}\right) / s_{0}^{\theta}\left(X_{j}\right)$ and $\tilde{R}_{n, 1}^{\theta}=n^{-1} \sum_{j=1}^{n} \rho_{n}\left(s_{n}^{\theta}\left(X_{j}\right)\right) d_{\theta}\left(X_{j}\right) H_{2, n}^{\theta}\left(X_{j}\right)$ $/ s_{0}^{\theta}\left(X_{j}\right)$. Again by the expansion of $d_{\theta}$ and that $\tilde{\xi}_{1}^{\theta}=\mathcal{O}\left(\delta_{n}\right)$, we have $B_{n, 2}^{\theta}=\mathcal{O}\left(h+\delta_{n}\right)$. It is easy to check that $H_{2, n}^{\theta}=\mathcal{O}\left(h \delta_{n}+\delta_{n}^{2}\right)$. We have

$$
\begin{aligned}
\tilde{R}_{n, 1}= & n^{-1} \sum_{j=1}^{n}\left[\rho_{n}\left(f_{\theta}\left(X_{j}\right)\right)+\rho_{n}^{\prime}\left(f_{\theta}\left(X_{j}\right)\right)\left\{f_{\theta}\left(X_{j}\right)+\frac{1}{2} f_{\theta}^{\prime \prime}\left(X_{j}\right) h^{2}+\epsilon_{0}^{\theta}\left(X_{j}\right)\right\}\right]\left\{g^{\prime}\left(\theta_{0}^{\top} X_{j}\right)+\frac{1}{6} g^{\prime \prime \prime}\left(\theta_{0}^{\top} X_{j}\right) h^{2}\right. \\
& \left.+\tilde{V}_{n}^{\theta}\left(X_{j}\right) / h\right\} H_{2, n}^{\theta}\left(X_{j}\right) f_{\theta}^{-1}\left(X_{j}\right)\left\{1-\frac{1}{2} f_{\theta}^{-1}\left(X_{j}\right) f_{\theta}^{\prime \prime}\left(X_{j}\right) h^{2}-f_{\theta}^{-1}\left(X_{j}\right) \epsilon_{0}^{\theta}\left(X_{j}\right)\right\}+\mathcal{O}\left(n^{2 \varsigma} \gamma_{n}^{3}\right) \\
& \stackrel{\text { def }}{=} R_{n, 1}+\mathcal{O}\left(n^{2 \varsigma} \gamma_{n}^{3}\right) .
\end{aligned}
$$

Next, we need to consider the terms in $R_{n, 1}$ one by one. Write

$$
\begin{aligned}
R_{n, 1,1}^{\theta} & \stackrel{\text { def }}{=} n^{-1} \sum_{j=1}^{n} \rho_{n}\left(f_{\theta}\left(X_{j}\right)\right) f_{\theta}^{-1}\left(X_{j}\right)\left(f_{\theta}\left(X_{j}\right) \nu_{\theta}\left(X_{j}\right)\right)^{\prime} e_{1}^{\theta} h \\
& =h n^{-2} \sum_{i=1}^{n}\left\{\sum_{j=1}^{n} K_{h}^{\theta}\left(X_{i j}\right) \rho_{n}\left(f_{\theta}\left(X_{j}\right)\right) f_{\theta}^{-1}\left(X_{j}\right)\left(f_{\theta}\left(X_{j}\right) \nu_{\theta}\left(X_{j}\right)\right)^{\prime}\right\} \varepsilon_{i} .
\end{aligned}
$$

Note that $E\left\{\rho_{n}\left(f_{\theta}(X)\right) f_{\theta}^{-1}(X)\left(f_{\theta}(X) \nu_{\theta}(X)\right)^{\prime} \mid \theta^{\top} X\right\}=0$. We have by Lemma 6.1

$$
R_{n, 1,1}^{\theta}=\mathcal{O}\left\{h n^{-1}\left(h^{-1} \log n\right)^{-1 / 2}\right\}
$$


and

$$
\begin{aligned}
E\left\{\mathcal{E}_{0}^{\theta} R_{n, 1,1}^{\theta}\right\} & =h n^{-3} E \sum_{i=1}^{n}\left\{\sum_{j=1}^{n} K_{h}^{\theta}\left(X_{i j}\right) \rho_{n}\left(f_{\theta}\left(X_{j}\right)\right) f_{\theta}^{-1}\left(X_{j}\right)\left(f_{\theta}\left(X_{j}\right) \nu_{\theta}\left(X_{j}\right)\right)^{\prime}\right\} \rho_{n}\left(f_{\theta}\left(X_{i}\right)\right) g^{\prime}\left(\theta^{\top} X_{i}\right) \nu_{\theta}\left(X_{i}\right) \varepsilon_{i}^{2} \\
& =h n^{-3} E\left\{\sum_{j=1}^{n} K_{h}^{\theta}(0) \rho_{n}\left(f_{\theta}\left(X_{j}\right)\right) f_{\theta}^{-1}\left(X_{j}\right)\left(f_{\theta}\left(X_{j}\right) \nu_{\theta}\left(X_{j}\right)\right)^{\prime} \rho_{n}\left(f_{\theta}\left(X_{j}\right)\right) g^{\prime}\left(\theta^{\top} X_{j}\right) \nu_{\theta}\left(X_{j}\right) \varepsilon_{j}^{2}\right\} \\
& =O\left(n^{-2}\right) .
\end{aligned}
$$

Applying similar approach to all the terms in $R_{n, 1}^{\theta}$, we have

$$
R_{n, 1}^{\theta}=\mathcal{O}\left\{n^{-1}(\log n / h)^{1 / 2}+(\log n / n)^{1 / 2} h^{2}\right\} \quad \text { and } \quad E\left\{\mathcal{E}_{n}^{\theta} R_{n, 1}^{\theta}\right\}=o\left\{(n h)^{-2}+h^{8}\right\}
$$

By Lemmas 5.1 and 6.1, we have

$$
B_{n, 3}^{\theta} \stackrel{\text { def }}{=} n^{-2} \sum_{j=1}^{n} \rho\left(s_{0}^{\theta}\left(X_{j}\right)\right) d_{\theta}\left(X_{j}\right) \sum_{i=1}^{n} K_{h}^{\theta}\left(X_{i j}\right) X_{i j}\left\{\tilde{B}^{\theta}\left(X_{j}, X_{i}\right)-B_{n}^{\theta}\left(X_{j}\right)\right\}^{\top} / s_{0}^{\theta}\left(X_{j}\right)=W_{n}^{\theta}+\mathcal{O}\left\{\left(\gamma_{n}+\delta_{\theta}\right) / h\right\} .
$$

By Lemma 5.2, we have

$$
n^{-2} \sum_{j=1}^{n} \rho\left(s_{0}^{\theta}\left(X_{j}\right)\right) d_{\theta}\left(X_{j}\right) \sum_{i=1}^{n} K_{h}^{\theta}\left(X_{i j}\right) X_{i j} \varepsilon_{i} / s_{0}^{\theta}\left(X_{j}\right)=\mathcal{E}_{0}^{\theta}+\frac{\tilde{c}_{1, n}}{n h}+B_{n, 4}^{\theta}\left(\theta_{0}-\theta\right)+R_{n, 2}^{\theta}+\mathcal{O}\left(n^{2 \varsigma} \gamma_{n}^{3}\right),
$$

where $\tilde{c}_{1, n}$ is defined in the lemma, and

$$
B_{4, n}^{\theta}=n^{-1} \sum_{j=1}^{n}\left\{\rho_{n}\left(f_{\theta}\left(X_{j}\right)\right)+\rho_{n}^{\prime}\left(f_{\theta}\left(X_{j}\right)\right) \epsilon_{0}^{\theta}\left(X_{j}\right)\right\} \eta_{n}^{\theta}\left(X_{j}\right)\left(\tilde{B}_{n}^{\theta}\left(X_{j}\right)\right)^{\top} / h
$$

and

$$
R_{n, 2}^{\theta}=n^{-1} \sum_{j=1}^{n}\left[\frac{1}{6} \rho_{n}\left(f_{\theta}\left(X_{j}\right)\right) g^{\prime \prime \prime}\left(\theta_{0}^{\top} X_{j}\right) h^{2}+\rho_{n}^{\prime}\left(f_{\theta}\left(X_{j}\right)\right) \epsilon_{0}^{\theta}\left(X_{j}\right)\left\{g^{\prime}\left(\theta_{0}^{\top} X_{j}\right)+\tilde{V}_{n}^{\theta}\left(X_{j}\right) / h\right\}\right] \eta_{n}^{\theta}\left(X_{j}\right) .
$$

Noting that $\eta_{n}^{\theta}=\mathcal{O}\left(\delta_{n}\right)$, we have $B_{4, n}^{\theta}=\mathcal{O}\left(\delta_{n} / h\right)$. Similarly, we have

$$
n^{-2} \sum_{j=1}^{n} \rho_{n}\left(s_{0}^{\theta}\left(X_{j}\right)\right) d_{\theta}\left(X_{j}\right) V_{n}^{\theta}\left(X_{j}\right) \sum_{i=1}^{n} K_{h}^{\theta}\left(X_{i j}\right) X_{i j} / s_{0}^{\theta}\left(X_{j}\right)=R_{n, 3}^{\theta}+\mathcal{O}\left(n^{2 \varsigma} \gamma_{n}^{3}\right),
$$

where

$$
R_{n, 3}^{\theta}=n^{-1} \sum_{j=1}^{n} \rho_{n}\left(s_{0}^{\theta}\left(X_{j}\right)\right) d_{\theta}\left(X_{j}\right) V_{n}^{\theta}\left(X_{j}\right)\left[\nu_{\theta}\left(X_{j}\right)+\frac{1}{2} f_{\theta}^{-1}\left\{\left(f_{\theta} \nu_{\theta}\right)^{\prime \prime}-f_{\theta}^{-1} f_{\theta}^{\prime \prime} \nu_{\theta}\left(X_{j}\right)\right\} h^{2}+\xi_{0}^{\theta}\left(X_{j}\right)-\epsilon_{0}^{\theta}\left(X_{j}\right)\right] .
$$

By the same arguments leading to (35), we have

$$
\begin{aligned}
& R_{n, 2}^{\theta}=\mathcal{O}\left\{n^{-1}(\log n / h)^{1 / 2}+(\log n / n)^{1 / 2} h^{2}\right\} \quad \text { and } \quad E\left\{\mathcal{E}_{n}^{\theta} R_{n, 2}^{\theta}\right\}=o\left\{(n h)^{-2}+h^{8}\right\} \\
& R_{n, 3}^{\theta}=\mathcal{O}\left\{n^{-1}(\log n / h)^{1 / 2}+(\log n / n)^{1 / 2} h^{2}\right\} \quad \text { and } \quad E\left\{\mathcal{E}_{n}^{\theta} R_{n, 3}^{\theta}\right\}=o\left\{(n h)^{-2}+h^{8}\right\}
\end{aligned}
$$


Lemma 5.4 follows from the above equations with $\mathcal{R}_{n}^{\theta}=R_{n, 1}^{\theta}+R_{n, 2}^{\theta}+R_{n, 3}^{\theta}$ and $\mathcal{B}_{n}^{\theta}=B_{n, 2}^{\theta}+B_{n, 3}^{\theta}+B_{n, 4}^{\theta}=$ $W_{n}^{\theta}+\mathcal{O}\left\{n^{2 \varsigma}\left(\gamma_{n}+\delta_{\theta}\right) / h\right\}$.

Acknowledgements The first author is most grateful to Professor V. Spokoiny for helpful discussions and NUS FRG R-155-000-048-112 and the Alexander von Humboldt Foundation for financial support. The second author thanks the Deutsche Forschungsgemeinschaft SFB 649 "Ökouomisches Risiko" for financial support. This paper was partly written while the third author was a Universidad Carlos III de Madrid-Banco Santander Chair of Excellence, and he thanks them for financial support.

\section{References}

[1] Bickel, P., Klaassen, A. J., Ritov, Y. and Wellner, J. A. (1993) Efficient and Adaptive Inference in Semiparametric Models, Baltimore: Johns Hopkins University Press.

[2] Carroll, R.J., Fan. J. Gijbels, I. and Wand, M.P. (1997) Generalized partially linear single-index models. J. Am. Statist. Ass., 92, 477-489.

[3] Delecroix, M., Hristache, M. and Patilea, V. (2004) On semiparametric M-estimation in single-index regression. J. Statist. Plann. and Infer. (to appear).

[4] Fan, J. and Gijbels, I. (1996) Local Polynomial Modeling and Its Applications. Chapman \& Hall, London.

[5] Fan, J. and Yao, Q. (2003) Nonlinear Time Series : nonparametric and parametric methods. New York : Springer Verlag.

[6] Friedman, J. H. (1984) SMART User's Guide. Laboratory for Computational Statistics, Stanford University Technical Report No. 1.

[7] Härdle, W., Hall, P. and Ichimura, H. (1993) Optimal smoothing in single-index models. Ann. Statist., 21, 157-178.

[8] Härdle, W. and Stoker, T. M. (1989) Investigating smooth multiple regression by method of average derivatives. J. Amer. Stat. Ass. 84 986-995.

[9] Härdle, W. and A.B. Tsybakov (1993). How sensitive are average derivatives? Journal of Econometrics $5831-48$.

[10] Horowitz, J.L. \& Härdle, W. (1996) Direct semiparametric estimation of single-index models with discrete covariates. J Amer. Stat. Assoc., 91 1632-1640. 
[11] Hristache, M., Juditsky, A. and Spokoiny, V. (2001) Direct estimation of the single-index coefficients in single-index models. Ann. Statist.

[12] Ichimura, H. (1993) Semiparametric least squares (SLS) and weighted SLS estimation of single-index models. J. Econometrics 58 71-120.

[13] Ichimura, H. and Lee, L. (1991) Semiparametric least squares estimation of multiple index models: Single equation estimation. Nonparametric and Semiparametric Methods in Econometrics and Statistics, edited by Barnett, W., Powell, J. and Tauchen, G.. Cambridge University Press.

[14] Li, K. C. (1991) Sliced inverse regression for dimension reduction (with discussion). Amer. Statist. Ass., 86, 316-342.

[15] Linton, O. (1995) Second order approximation in the partially linear regression model. Econometrica, 63, 1079-1112.

[16] Nishiyama, Y., and P. M. Robinson (2000). Edgeworth expansions for semiparametric average derivatives. Econometrica 68, 931-980.

[17] Nishiyama, Y., and P. M. Robinson (2005). The Bootstrap and the Edgeworth Correction for semiparametric average derivatives. Econometrica 73, 903-948.

[18] Penrose, R. (1955) A generalized inverse for matrices, Proc. Cambridge Philos. Soc. 51, 406-413.

[19] Powell, J.L., J.H. Stock, and T.M. Stoker (1989). Semiparametric estimation of index coefficients. Econometrica 57, 1403-1430.

[20] Powell, J.L. and T.M. Stoker (1996). Optimal bandwidth choice for density weighted averages. Journal of Econometrics 755, 291-316.

[21] Ruppert, D., Sheather, J., and Wand, P. M. (1995) An effective bandwidth selector for local least squares regression. J. Am. Statist. Ass., 90, 1257-1270.

[22] Schott, J.R. (1997) Matrix Analysis for Statistics. John Wiley \& Sons. New York.

[23] Weisberg, S. and Welsh, A. H. (1994) Estimating the missing link functions, Ann. of Statist. 22, 16741700. 
[24] Xia, Y., Tong, H., Li, W. K. and Zhu, L. (2002) An adaptive estimation of dimension reduction space (with discussions). J. Roy. Statist. Soc. B., 64, 363-410.

[25] Xia, Y. (2006). Asymptotic distributions for two estimators of the single-index model. Econometric Theory (to appear)

[26] Xia, Y. and Li, W. K. (1999) On single-index coefficient regression models. Journal of the American Statistical Association, 94, 1275-1285.

[27] Yin, X. \& Cook, R. D. (2005). Direction estimation in single-index regressions. Biometrika, 92, 371-384. 